\title{
CROSS EFFECTS AND CALCULUS IN AN UNBASED SETTING
}

\author{
KRISTINE BAUER, BRENDA JOHNSON, AND RANDY MCCARTHY, \\ WITH AN APPENDIX BY ROSONA ELDRED
}

\begin{abstract}
We study functors $F: \mathcal{C}_{f} \rightarrow \mathcal{D}$ where $\mathcal{C}$ and $\mathcal{D}$ are simplicial model categories and $\mathcal{C}_{f}$ is the category consisting of objects that factor a fixed morphism $f: A \rightarrow B$ in $\mathcal{C}$. We define the analogs of Eilenberg and Mac Lane's cross effect functors in this context, and identify explicit adjoint pairs of functors whose associated cotriples are the diagonals of the cross effects. With this, we generalize the cotriple Taylor tower construction of Deriving calculus with cotriples (by the second and third authors) from the setting of functors from pointed categories to abelian categories to that of functors from $\mathcal{C}_{f}$ to $\mathcal{S}$, a suitable category of spectra, to produce a tower of functors $\cdots \rightarrow \Gamma_{n+1} F \rightarrow \Gamma_{n} F \rightarrow \Gamma_{n-1} F \rightarrow \cdots \rightarrow F(B)$ whose $n$th term is a degree $n$ functor. We compare this tower to Goodwillie's tower, $\cdots \rightarrow P_{n+1} F \rightarrow P_{n} F \rightarrow P_{n-1} F \rightarrow \cdots \rightarrow F(B)$, of $n$-excisive approximations to $F$ found in his work Calculus II. When $F$ is a functor that commutes with realizations, the towers agree. More generally, for functors that do not commute with realizations, we show that the terms of the towers agree when evaluated at the initial object of $\mathcal{C}_{f}$.
\end{abstract}

\section{INTRODUCTION}

Tom Goodwillie's calculus of homotopy functors is a technique for studying homotopy functors of spaces and spectra $(9,10])$. It provides a means by which a homotopy functor can be approximated by an $n$-excisive functor in a manner analogous to the degree $n$ Taylor polynomial approximation of a real-valued function. Because of this analogy, the sequence of approximating functors, $P_{1} F, P_{2} F, \ldots$, $P_{n} F, \ldots$, associated to a functor $F$ by Goodwillie's method is referred to as the Taylor tower of $F$. In the decades since its initial development, Goodwillie's theory has been further developed and applied by many other mathematicians.

In an effort to apply the calculus of functors to a more algebraic setting and to better understand the combinatorics underlying Goodwillie's constructions, the second and third authors of this paper developed a model for a Taylor tower for functors of abelian categories based on a particular collection of cotriples arising from Eilenberg and Mac Lane's cross effect functors ([13]). For a functor $F$, the terms in the resulting sequence of approximations, $\left\{\Gamma_{n} F\right\}$, behave in a slightly different way than the $n$-excisive approximations provided by Goodwillie. Goodwillie's functors $P_{n} F$ satisfy a higher-order excision property, while the polynomial functors $\Gamma_{n} F$ satisfy a kind of higher additivity property. The cotriple model for calculus has the advantage that the difference between a homotopy functor $F$ and its polynomial approximation $\Gamma_{n} F$ can be modeled by cotriple homology, which

Received by the editors January 5, 2011 and, in revised form, June 29, 2012, February 6, 2013, April 11, 2013 and February 28, 2014.

2010 Mathematics Subject Classification. Primary 55P65; Secondary 18G55, 18G30. 
is well understood. Furthermore, in good situations the cotriple method recovers information about Goodwillie's functors. In particular, if $\mathcal{C}$ is the category of based topological spaces, and $F: \mathcal{C} \rightarrow \mathcal{C}$ is a homotopy functor taking values in connected spaces that commutes with geometric realization, Andrew Mauer-Oats generalized the cotriple method and showed that $P_{n} F \simeq \Gamma_{n} F$ ([16], [17]).

The cotriple method as established in 13 is limited; it only applies to functors $F: \mathcal{C} \rightarrow \mathcal{D}$, where $\mathcal{C}$ is a pointed category (a category with an object that is both initial and final) with finite coproducts and $\mathcal{D}$ is an abelian category. The generalization of the cotriple method to the topological setting in [16] and [17] is similarly limited as it applies to functors of based spaces. On the other hand, Goodwillie's construction can be used for functors whose source categories are not pointed and whose target categories are not abelian, in particular, functors from the category Top of unbased topological spaces or $T o p / Y$ of topological spaces over a fixed space $Y$ to categories of spaces or spectra.

In [13, the essential cotriples are obtained by identifying adjoint pairs of functors for which the right adjoint is a cross effect functor. Goodwillie ([10]) identifies a similar adjunction up to homotopy in the topological setting of (not necessarily basepointed) spaces and spectra. Working with basepointed spaces, Mauer-Oats ([16]) shows directly that diagonals of cross effect functors form the cotriples in which we are interested, but does not identify the adjoint pairs from which these cotriples arise. This suggests that there should be some adjoint pairs of functors that generate the cotriples in the topological setting, at least when the objects are basepointed. A key result in the present paper is to show that this is true for fairly general model categories, even in the unpointed case. As an application of the cotriples that one obtains from these strict adjoint pairs, we generalize the construction of the Taylor tower in [13] and obtain analogous results, including a variation of Mauer-Oats' result that relates the terms in the cotriple Taylor tower to those of Goodwillie's. We summarize the main results of the paper below.

We work with functors $F: \mathcal{C}_{f} \rightarrow \mathcal{D}$, where $\mathcal{C}$ and $\mathcal{D}$ are simplicial model categories and $\mathcal{C}_{f}$ is the category that consists of objects $A \rightarrow X \rightarrow B$ factoring a fixed morphism $f: A \rightarrow B$ in $\mathcal{C}$. In [13, we used an adjoint pair involving the $n$th cross effect functor to define a cotriple $\perp_{n}$ on the category of functors from a pointed category with finite coproducts to an abelian category. The cotriple $\perp_{n}$ yielded the $(n-1)$ st term in our Taylor tower. The main difficulty in reconstructing the cotriple $\perp_{n}$ in the category of functors from $\mathcal{C}_{f}$ to $\mathcal{D}$ is that the pair of functors used in [13] is no longer an adjoint pair, but instead only gives us an adjunction up to homotopy. We resolve this issue by factoring through a category of coalgebras associated to a certain cotriple $t$ to obtain a pair of adjunctions whose composition produces the adjunction we need. This gives us the following result. The functor $\perp_{n}$ is the diagonal of the $n$th cross effect.

Theorem 3.8, Theorem 3.14, Theorem 3.17. For each $n \geq 1$, there is a cotriple $t$ on the category of functors of $n$ variables from $\mathcal{C}_{f}$ to $\mathcal{D}$, and an adjoint pair of functors $\left(U^{+}, t^{+}\right)$between this category and the category of $t$-coalgebras, where the forgetful functor $U^{+}$is the left adjoint. There is a second adjoint pair of functors $\left(\Delta^{*}, \sqcup_{n}\right)$ between the category of functors of $n$ variables from $\mathcal{C}_{f}$ to $\mathcal{D}$ and the category of functors of a single variable from $\mathcal{C}_{f}$ to $\mathcal{D}$, with the diagonal functor $\Delta^{*}$ as the left adjoint. The composition yields the adjoint pair $\left(\Delta^{*} \circ U^{+}, t^{+} \circ \sqcup_{n}\right)$ whose associated cotriple is $\perp_{n}$, defined on the category of functors from $\mathcal{C}_{f}$ to $\mathcal{D}$. 
We can use the cotriples of Theorem 3.17 as the basis for constructing terms in a Taylor tower for $F: \mathcal{C}_{f} \rightarrow \mathcal{S}$, where $\mathcal{S}$ is a suitable category of spectra. However, the $n$th term in this tower, $\Gamma_{n} F$, is not an $n$-excisive functor as Goodwillie constructs, but instead a degree $n$ functor. A functor is degree $n$ if its $(n+1)$ st cross effect vanishes, whereas a functor is $n$-excisive if it takes strongly cocartesian $(n+1)$ cubical diagrams of objects (i.e., diagrams whose square faces are all homotopy pushouts) to homotopy pullback diagrams. We compare the notions of $n$-excisive and degree $n$, proving that being degree $n$ is a weaker condition that can yield $n$-excisive behavior in certain circumstances. When $F$ commutes with realizations, we prove that the notions of degree $n$ and $n$-excisive coincide, and that the functors $P_{n} F$ and $\Gamma_{n} F$ agree. In particular, we have the following results.

Proposition 4.11, If $F: \mathcal{C}_{f} \rightarrow \mathcal{S}$ commutes with realizations, then $F$ is degree $n$ if and only if $F$ is n-excisive.

We use Proposition 4.11 to obtain a Mauer-Oats style result, showing that there is a fibration sequence of functors involving $P_{n} F$ and $\perp_{n+1}^{*+1} F$, the simplicial object associated to the cotriple $\perp_{n+1}$ and functor $F$.

Theorem 6.5. Let $F: \mathcal{C}_{f} \rightarrow \mathcal{S}$ be a functor that commutes with realizations. Then there is a (co)fibration sequence of functors

$$
\left|\perp_{n+1}^{*+1} F\right| \rightarrow F \rightarrow P_{n} F .
$$

As a consequence of Theorem 6.5, we obtain

Corollary 6.8. Let $F: \mathcal{C}_{f} \rightarrow \mathcal{S}$ be a functor that commutes with realizations. Then $P_{n} F$ and $\Gamma_{n} F$ are weakly equivalent as functors from $\mathcal{C}_{f}$ to $\mathcal{S}$.

When $F$ does not commute with realizations, the role of the initial object, $A$, in $\mathcal{C}_{f}$ becomes more critical in comparing the notions of degree $n$ and $n$-excisive, and in comparing $\Gamma_{n} F$ and $P_{n} F$. We say that a functor is $n$-excisive relative to $A$ if it behaves like an $n$-excisive functor on strongly cocartesian $(n+1)$-cubical diagrams whose initial objects are $A$, and prove

Proposition 4.3. Let $F$ be a functor from $\mathcal{C}_{f}$ to $\mathcal{S}$. Let $n \geq 1$ be an integer. The functor $F$ is degree $n$ if and only if $F$ is n-excisive relative to $A$.

We also prove that the results of Theorem 6.5 and Corollary 6.8 apply when the functors are evaluated at $A$. In particular, we have

Theorem 6.9. Let $F: \mathcal{C}_{f} \rightarrow \mathcal{S}$, where $\mathcal{C}_{f}$ is the category of objects factoring the morphism $f: A \rightarrow B$. Then $\Gamma_{n} F(A) \simeq P_{n} F(A)$.

An important realization is that Theorem 6.9 can be rephrased to show that for any $X$, the $n$th term of Goodwillie's tower can be recovered from the $n$th term in some cotriple Taylor tower, even though the towers do not agree as functors. To do so, we change our focus to the category of objects over a fixed terminal object $B$. This focus on the terminal object is exactly the same as the setting in [10]. Let $\mathcal{C}_{/ B}$ be the category of objects in $\mathcal{C}$ over $B$, and let $F$ be a functor from $\mathcal{C}_{/ B}$ to spectra. Given any $\beta: X \rightarrow B$ in $\mathcal{C}_{/ B}$, we have a weak equivalence of spectra

$$
P_{n} F(X) \simeq \Gamma_{n}^{\beta} F(X),
$$

where $\left\{\Gamma_{n}^{\beta} F\right\}$ is the cotriple Taylor tower obtained by restricting $F$ to the category $\mathcal{C}_{\beta}$. 
A key step in proving Theorem 6.9 is the observation below. The functor $T_{n} F$ is the first stage in the sequence of functors that Goodwillie uses to construct $P_{n} F$.

Lemma 6.10, Let $F: \mathcal{C}_{f} \rightarrow \mathcal{S}$. Then

$$
\perp_{n+1} F(A) \rightarrow F(A) \rightarrow T_{n} F(A)
$$

is a fibration sequence in $\mathcal{S}$.

As an appendix, we include a generalization of this result due to Rosona Eldred:

Proposition B.1. For a functor $F: \mathcal{C}_{f} \rightarrow \mathcal{S},\left|\operatorname{sk}_{k}\left(\perp_{n+1}^{*+1} F\right)(A)\right| \rightarrow F(A) \rightarrow$ $T_{n}^{k+1} F(A)$ is a homotopy fiber sequence where $\mathrm{sk}_{k}$ denotes the $k$-skeleton of the simplicial object $\perp_{n+1}^{*+1} F$.

The paper is organized as follows. In section 2 we take care of preliminaries: we define the types of categories in which we will be working, describe the models for and properties of homotopy limits and colimits that we use, and review some basic notions associated to $n$-cubical diagrams of objects in our categories. In section 3 we define cross effects for functors from $\mathcal{C}_{f}$ to $\mathcal{D}$. We also identify the composition of adjoint pairs that yields $\perp_{n}$ as a cotriple. In section 4 , notions of degree $n$ and $n$-excisive are compared via the intermediate concept of $n$-excisive relative to $A$. In section 5 , the cotriple Taylor tower is defined and various properties are verified for it. This leads to a comparison in section 6 of the cotriple Taylor tower in this context with Goodwillie's tower.

\section{PreRequisites}

In this section we describe the context in which we will be working, and review some essential concepts that will be used throughout this paper. The section is divided into three parts. The first describes the categories with which we work and provides a summary of some properties of model categories, simplicial model categories, and categories of simplicial objects that we need. The second covers necessary facts about homotopy limits and colimits. The third discusses $n$-cubical diagrams.

2.1. The setting. We work with functors from $\mathcal{C}$ to $\mathcal{D}$, where $\mathcal{C}$ and $\mathcal{D}$ are suitable model categories. By suitable, we mean that $\mathcal{C}$ and $\mathcal{D}$ should be simplicial model categories, that $\mathcal{C}$ has a functorial cofibrant replacement functor and that $\mathcal{D}$ has a functorial fibrant replacement functor. For many results, we will also require that $\mathcal{D}$ (but not $\mathcal{C}$ ) be pointed, i.e., that it has an object that is both initial and final. Recall that a model category comes equipped with distinguished classes of morphisms weak equivalences, cofibrations, and fibrations - satisfying the standard axioms (as found on pp. 1.1-1.2 of [18, or in several expository accounts, such as Definition 1.3 of [8]). Requiring that a category $\mathcal{D}$ be a simplicial model category gives us the following extra structure:

- for every simplicial set $K$ and object $X$ of $\mathcal{D}$ there is an object $X \otimes K$ in $\mathcal{D}$; and

- for every simplicial set $K$ and object $Y$ of $\mathcal{D}$ there is an exponential object $Y^{K}$ in $\mathcal{D}$ defined by the adjunction formula

$$
\operatorname{hom}_{\mathcal{D}}(X \otimes K, Y) \cong \operatorname{hom}_{\mathcal{D}}\left(X, Y^{K}\right) ;
$$


- for each pair of objects $X$ and $Y$, there is a simplicial set of morphisms in $\mathcal{D}, \operatorname{Hom}_{\mathcal{D}}(X, Y)$, satisfying an additional axiom (see pp. 1.1, 1.2, and 2.2 of 18 ).

For much of this paper we focus on subcategories of $\mathcal{C}$ determined by morphisms in $\mathcal{C}$. In particular, for a morphism $f: A \rightarrow B$ in $\mathcal{C}$, the category $\mathcal{C}_{f}$ is the category whose objects are pairs of morphisms in $\mathcal{C}$ of the form $A \rightarrow X \rightarrow B$ that provide a factorization of $f: A \rightarrow B$. We will usually denote objects of $\mathcal{C}_{f}$ simply by the object $X$ through which $f$ factors. A morphism in $\mathcal{C}_{f}$ is a commuting diagram:

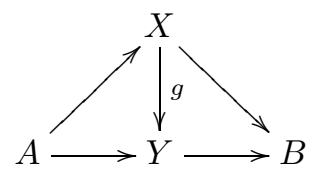

which we will denote as $g: X \rightarrow Y$ when the context is clear. The category $\mathcal{C}_{f}$ has initial object $A=A \rightarrow B$ (the first map is the identity and the second map is $f$ ) and terminal object $A \rightarrow B=B$ (the first map is $f$ and the second map is the identity).

The category $\mathcal{C}_{f}$ inherits structure from $\mathcal{C}$. More specifically, it is a simplicial model category whenever $\mathcal{C}$ is [18, §II.2, Proposition 6]. The map $g$ in $\mathcal{C}_{f}$ is a weak equivalence if the underlying map $g: X \rightarrow Y$ is a weak equivalence in $\mathcal{C}$, a cofibration in $\mathcal{C}_{f}$ if the underlying map in $\mathcal{C}$ is a cofibration, and a fibration if $g$ is a fibration in $\mathcal{C}$. For convenience, we assume from the outset that all objects of $\mathcal{C}_{f}$ in this paper are cofibrant. That is, we assume that an object $X$ of $\mathcal{C}_{f}$ is a factorization $A \longmapsto X \rightarrow B$ of $f$ where the map $A \longmapsto B$ is a cofibration in $\mathcal{C}$. Since we assume all objects are cofibrant, we abuse notation and simply denote the category of cofibrant objects by $\mathcal{C}_{f}$. Limits and colimits in $\mathcal{C}_{f}$ are also inherited from $\mathcal{C}$, i.e., they can be computed in the underlying category $\mathcal{C}$.

We will occasionally pass to the category $s \mathcal{C}_{f}$ of simplicial objects in $\mathcal{C}_{f}$. When doing so, we extend the model category structure of $\mathcal{C}_{f}$ to $s \mathcal{C}_{f}$ using the Reedy model structure. Recall that to do so, one uses a Quillen pair. The following definition dates to [18] and can be found in many modern references, e.g., [8].

Definition 2.1. Let $\mathcal{C}$ and $\mathcal{D}$ be model categories and

$$
F: \mathcal{C} \longleftrightarrow \mathcal{D}: G
$$

be an adjoint pair of functors, with $F: \mathcal{C} \rightarrow \mathcal{D}$ the left adjoint. Then $F$ and $G$ are called a Quillen pair (or Quillen functor) if

- $F$ preserves cofibrations and weak equivalences between cofibrant objects and

- $G$ preserves fibrations and weak equivalences between fibrant objects.

We would like to produce an adjoint pair of functors between $\mathcal{C}_{f}$ and $s \mathcal{C}_{f}$ which becomes a Quillen pair when we put the correct model structure on $s \mathcal{C}_{f}$. The left adjoint of the (potential) Quillen pair is the geometric realization functor. Recall that $\Delta$ is the category whose objects are ordered sets $[n]=\{0,1,2, \ldots, n\}$ for $n \geq 0$ and morphisms are order-preserving set maps. For $m \geq 0$, the standard $m$-simplex is $\Delta^{m}=\operatorname{hom}_{\Delta}(-,[m])$. If $\mathcal{C}$ (and hence $\mathcal{C}_{f}$ ) is a simplicial model category and $X$. is a simplicial object over $\mathcal{C}_{f}$, then the object $\Delta^{m} \otimes X_{n}$ is well-defined for each $m, n \geq 0$. The geometric realization of $X$. in $s \mathcal{C}_{f}$, denoted $|X$.$| , is the coequalizer$ 
of

$$
\coprod_{[m] \rightarrow[n]} \Delta^{m} \otimes X_{n} \Longrightarrow \coprod_{n} \Delta^{n} \otimes X_{n},
$$

where the first coproduct runs over all possible morphisms from $[m]$ to $[n]$ in $\Delta$ and the two arrows correspond to evaluation on $\Delta^{m}$ and $X_{n}$, respectively. Its right adjoint is the singular simplicial set functor. The singular simplicial set of an object $Y$ is the simplicial object $Y .^{\Delta}$ defined by $\left(Y^{\Delta}\right)_{n}=Y^{\Delta^{n}}$, with face and degeneracy maps induced by the ones in $\Delta$.

Placing the Reedy model category structure on $s \mathcal{C}_{f}$ guarantees that the geometric realization and the singular simplicial set functors are a Quillen pair. The cofibrations and fibrations for this structure can be readily described via latching and matching objects. The $n$th latching object consists of the degenerate simplices in the degree $n$ part of a simplicial object. If $X \in s_{f}$, then

$$
L_{n} X .:=\left(\mathrm{sk}_{n-1} X\right)_{n}=\underset{\phi:[n] \rightarrow[m]}{\operatorname{colim}} \phi^{*} X_{m}
$$

where the colimit is taken over all surjections from $[n]$ in $\Delta$. Note that the degeneracy maps of $X$. provide a (natural) map $L_{n} X \rightarrow X_{n}$. The $n$th matching object is defined similarly,

$$
M_{n} X:=\lim _{\phi:[m] \hookrightarrow[n]} \phi^{*} X_{m},
$$

where the limit is now taken over injections. There is a natural map $X_{n} \rightarrow M_{n} X$ that comes from the face maps of $X$.. The next theorem describes the Reedy structure and establishes that it gives us the desired model category structure.

Theorem $2.2([19])$. There is a model category structure on $s_{f}$ where a morphism $g: X . \rightarrow Y$. is

- a weak equivalence if $X_{n} \rightarrow Y_{n}$ is a weak equivalence in $\mathcal{C}_{f}$ for all $n \geq 0$;

- a cofibration if the natural morphism

$$
X_{n}+{ }_{L_{n} X} L_{n} Y:=\operatorname{colim}\left(X_{n} \leftarrow L_{n} X \rightarrow L_{n} Y\right) \rightarrow Y_{n}
$$

is a cofibration in $\mathcal{C}_{f}$ for all $n \geq 0$; and

- a fibration if the natural morphism

$$
X_{n} \rightarrow Y_{n} \times{ }_{M_{n} Y} M_{n} X:=\lim \left(M_{n} X \rightarrow M_{n} Y \leftarrow Y_{n}\right)
$$

is a fibration in $\mathcal{C}_{f}$ for all $n \geq 0$.

With this model category structure, the geometric realization and the singular simplicial set functors form a Quillen pair.

2.2. Homotopy limits and colimits. We use homotopy limits and colimits to describe certain desirable properties of our functors. Homotopy limits and colimits can be defined abstractly as total derived functors or, depending on the category, concretely in terms of specific models. There are several models for homotopy limits and colimits, typically involving a simplicial construction coming from the nerve of the underlying diagram category $I$. We describe the two particular models that we use in this paper and the properties of these models that we will need. For more details, we refer readers to [20, which provides a good expository account of homotopy limits and colimits in model categories, [9, which establishes many of the properties we use for topological spaces and spectra, [11] for more details about the model we use for homotopy limit or [1, the classical reference. The homotopy 
colimit model is that of [20], whereas the homotopy limit model is essentially the one described in [1, 9], and [1].

To construct homotopy colimits, we use the generalized bar construction of [20]. Let $\mathcal{X}: I \rightarrow \mathcal{C}_{f}$ be a functor. Then

$$
\operatorname{hocolim}_{I} \mathcal{X} \simeq|B \bullet(I, \mathcal{X})|,
$$

where the right hand side is the geometric realization of the simplicial object $B_{\bullet}(I, \mathcal{X})$ with

$$
B_{n}(I, \mathcal{X})=\coprod_{i_{0} \rightarrow \cdots \rightarrow i_{n}} \mathcal{X}\left(i_{0}\right) .
$$

The coproduct is indexed by the $n$-simplices of $N_{\bullet}(I)$, the nerve of $I$, and the face and degeneracy maps are given by those in $N_{\bullet}(I)$.

The following properties of $B_{\bullet}(I, \mathcal{X})$ are used in section 4 .

Lemma 2.3. If $\mathcal{X}$ is objectwise cofibrant, then

(1) $B_{\bullet}(I, \mathcal{X})$ is cofibrant under the Reedy model structure on s. $\mathcal{C}_{f}$.

(2) For any full subcategory $I^{\prime}$ of $I$, the induced map

$$
B_{\bullet}\left(I^{\prime}, \mathcal{X}\right) \rightarrow B_{\bullet}(I, \mathcal{X})
$$

is a cofibration in the Reedy model structure.

(3) If $N_{\bullet}(I)=N_{\bullet}\left(I^{\prime}\right) \cup N_{\bullet}\left(I^{\prime \prime}\right)$, then

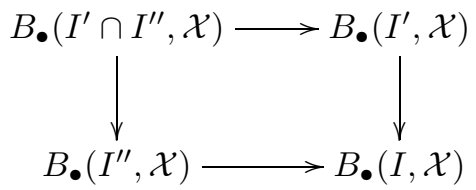

is a pushout diagram.

Proof. The first statement (1) is Lemma 9.2 of [20].

To prove (2), we must show that the map

$$
j: B_{n}\left(I^{\prime}, \mathcal{X}\right) \coprod_{L_{n} B_{\bullet}\left(I^{\prime}, \mathcal{X}\right)} L_{n} B_{\bullet}(I, \mathcal{X}) \rightarrow B_{n}(I, \mathcal{X})
$$

is a cofibration in $\mathcal{C}_{f}$. Note that $L_{n} B_{\bullet}(I, \mathcal{X})$ is the coproduct

$$
\coprod_{i_{0} \rightarrow \cdots \rightarrow i_{n}} \mathcal{X}\left(i_{0}\right)
$$

indexed by chains of maps $i_{0} \rightarrow \cdots \rightarrow i_{n}$ in $I$ for which some $i_{k} \rightarrow i_{k+1}$ is the identity map. The structure maps $L_{n} B_{\bullet}(I, \mathcal{X}) \rightarrow B_{n}(I, \mathcal{X})$ and $B_{n}\left(I^{\prime}, \mathcal{X}\right) \rightarrow$ $B_{n}(I, \mathcal{X})$ are induced by inclusion maps: the first is the inclusion of the degenerate elements, while the second is induced by the forgetful functor from $I^{\prime}$ to $I$. These inclusions mean that the map $j$ is constructed from maps that are cofibrations. That is, each summand $\mathcal{X}\left(i_{0}\right)$ in

$$
B_{n}\left(I^{\prime}, \mathcal{X}\right) \coprod_{L_{n} B \bullet\left(I^{\prime}, \mathcal{X}\right)} L_{n} B_{\bullet}(I, \mathcal{X})
$$

is indexed by a chain of maps $i_{0} \rightarrow \cdots \rightarrow i_{n}$ either in $I^{\prime}$, which is a subcategory of $I$, or else in $I$ itself (coming from a degenerate chain of maps in $I$ ), or both. Thus, $\mathcal{X}\left(i_{0}\right)$ also represents a summand of $B_{n}(I, \mathcal{X})$. To complete the construction of $j$, we take the coproduct of identity maps, one for each summand $\mathcal{X}\left(i_{0}\right)$ 
of $B_{n}\left(I^{\prime}, \mathcal{X}\right) \coprod_{L_{n} B \cdot\left(I^{\prime}, \mathcal{X}\right)} L_{n} B_{\bullet}(I, \mathcal{X})$ together with the coproduct over the initial object $A$ of maps $A \rightarrow \mathcal{X}\left(i_{0}\right)$ for each summand $\mathcal{X}\left(i_{0}\right)$ of $B_{n}(I, \mathcal{X})$ indexed by a chain of maps $i_{0} \rightarrow \cdots \rightarrow i_{n}$ which is neither degenerate nor contained in the subcategory $I^{\prime}$. Since $\mathcal{X}\left(i_{0}\right)$ is cofibrant, each of the maps $A \rightarrow \mathcal{X}\left(i_{0}\right)$ is a cofibration and the identity map is always a cofibration. The coproduct of cofibrations is again a cofibration, so it follows that $j$ is a cofibration.

For $(3)$, let $F\left(i_{0}\right)$ be a simplex of $B_{\bullet}(I, \mathcal{X})$ indexed by $i_{0} \rightarrow \cdots \rightarrow i_{n}$. Since $i_{0} \rightarrow \cdots \rightarrow i_{n}$ is a simplex of $N_{\bullet}(I)$, the hypothesis implies that $i_{0} \rightarrow \cdots \rightarrow i_{n}$ is a chain of morphisms in either $I^{\prime}$ or $I^{\prime \prime}$. Thus the simplex $F\left(i_{0}\right)$ came from one in $B_{\bullet}\left(I^{\prime}, \mathcal{X}\right)$ or $B_{\bullet}\left(I^{\prime \prime}, \mathcal{X}\right)$. Hence the diagram of $(3)$ is a pushout.

Convention. Given a simplicial model category $\mathcal{M}$, the simplicial realization preserves weak equivalences between cofibrant simplicial objects. If $\mathcal{M}$ has functorial cofibrant replacements, then one can define hocolim $\Delta^{o p}$ as a weak-equivalencepreserving functor from simplicial objects in $\mathcal{M}$ to $\mathcal{M}$ by composing the cofibrant replacement with a model for hocolim such as the one in [11, 18.1.2]. The functor hocolim $\triangle^{o p}$ is sometimes called the "fat" realization, and is written $\|X\|=$ hocolim $_{\triangle \text { op }} X$. There is a natural transformation $\|X\| \rightarrow|X|$ which is a weak equivalence when $X$ is a cofibrant simplicial object in $\mathcal{M}$. We will be using the fat realization throughout. We will follow the convention (which is a slight abuse of notation) and simply write $|X|$ for the fat realization (as was done in [17]). We note that by Lemma 2.3(1), when $\mathcal{X}$ is objectwise cofibrant, we can apply the fat realization to the diagram in Lemma $2.3(3)$ and obtain a cocartesian diagram.

The model for homotopy colimits described above can be dualized to produce a model for homotopy limits. Instead, we construct homotopy limits in the following fashion.

Definition 2.4. Let $I$ be a small category and $F: I \rightarrow \mathcal{C}$ be an $I$-diagram in $\mathcal{C}$. For each object $i$ in our indexing category $I$, let $I \downarrow i$ denote the category of elements in $I$ over $i$; this category has objects $j \rightarrow i$ and morphisms given by commuting triangles. Let $N_{\bullet}(I \downarrow i)$ be the nerve of the category $I \downarrow i$. Then

$$
\operatorname{holim}_{I} F=\operatorname{hom}^{I}\left(N_{\bullet}(I \downarrow-), F(-)\right),
$$

where for a functor $G$ from $I$ to s.set (simplicial sets) and a functor $H: I \rightarrow \mathcal{C}$, the construction $\operatorname{hom}^{I}(G, H)$ is the equalizer of the two obvious maps

$$
\prod_{i \in I} H(i)^{G(i)} \Longrightarrow \prod_{i \rightarrow j} H(j)^{G(i)}
$$

We make use of the following properties of homotopy limits. The first four are essential for the key results in section 3. We include their proofs in Appendix A. The last property follows easily from the definition of homotopy limit above.

Lemma 2.5. Let $I$ and $J$ be small categories.

(1) If $F: I \times J \rightarrow \mathcal{C}$, then

$$
\operatorname{holim}_{I \times J} F \cong \operatorname{holim}_{I}\left(\operatorname{holim}_{J} F\right) .
$$

(2) If $\alpha: J \rightarrow I$ and $F: I \rightarrow \mathcal{C}$, then there is a morphism $\operatorname{holim}_{I} F \rightarrow$ $\operatorname{holim}_{J} F \circ \alpha$. 
(3) If $T$ is a constant $I$-diagram with $T(C)=T$ and $T(f)=\mathrm{id}_{T}$, where $T$ is a terminal object of $\mathcal{C}$, then

$$
\operatorname{holim}_{I} T \cong T .
$$

(4) If $\mathcal{I}$ is the trivial category on $i$, then for any $\mathcal{I}$-diagram $X$,

$$
\operatorname{holim}_{\mathcal{I}} X \cong X(i) \text {. }
$$

(5) If $F, G: I \rightarrow \mathcal{C}$ and $\eta: F \rightarrow G$ is a natural transformation (also called a map of $I$-diagrams), then $\eta$ induces a map from $\operatorname{holim}_{I} F \rightarrow \operatorname{holim}_{I} G$.

As noted on page 379 of [11, the definition of homotopy limit above is homotopy invariant only when the diagram is objectwise fibrant. When the $I$-diagram $F$ is objectwise fibrant, $\operatorname{holim}_{I} F$ is fibrant by Corollary 18.5.2(2) of [11]. For this reason, we will use functors that take values in fibrant objects.

The first property of Lemma 2.5 is often referred to by the slogan "homotopy limits commute", since it also implies that holim holim $_{J}=$ holim $_{J}$ holim $_{I}$. A special case of this property tells us that homotopy fibers and homotopy limits commute, where homotopy fibers are defined as follows.

Definition 2.6. Let $\mathcal{D}$ be a pointed model category with initial/final object $\star$, and let $g: X \rightarrow Y$ be a morphism in $\mathcal{D}$. Then the homotopy fiber of $g$, denoted hofiber $g$, is the homotopy limit of

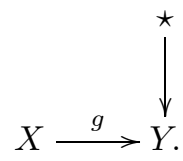

Note that in this paper, all homotopy fibers are computed in the target category $\mathcal{D}$.

2.3. Cubical diagrams. Later in this paper, we examine two fundamental concepts, degree $n$ and $n$-excisive, each of which is used to define a notion of degree $n$ polynomial functor. Both concepts are determined by the behavior of a functor when applied to certain types of diagrams in $\mathcal{C}_{f}$.

Definition 2.7. Let $\mathbf{n}=\{1,2, \ldots, n\}$ and let $\mathcal{P}(\mathbf{n})$ be the power set of $\mathbf{n}$ treated as a category whose objects are the subsets of $\mathbf{n}$ and morphisms are the set inclusions. An $n$-cubical diagram (or $n$-cube) in a category $\mathcal{D}$ is a functor from $\mathcal{P}(\mathbf{n})$ to $\mathcal{D}$.

One can picture an $n$-cubical diagram as being shaped like a cube of dimension $n$. For this reason, we say that the object $\chi(S) \in \mathcal{C}$ for any fixed $S \subset \mathbf{n}$ is a vertex of the $n$-cube $\chi$. Similarly, the image of the inclusion $S \subset S \cup\{i\}(1 \leq i \leq n, i \notin S)$ under $\chi$ is called an edge, and for $i, j \notin S$, the image of

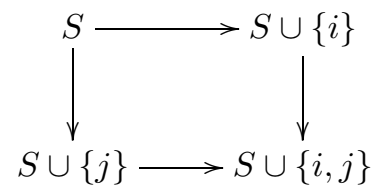

under $\chi$ is a 2 -face. To a functor of $n$ variables from $\mathcal{C}_{f}$, we associate two special $n$-cubical diagrams. 
Example 2.8. In the category $\mathcal{C}_{f}$, every object $X$ is equipped with a map $\beta_{X}$ to the terminal object $B$. Let $H: \mathcal{C}_{f}^{\times n} \rightarrow \mathcal{D}$ be a functor of $n$ variables from $\mathcal{C}_{f}$ to an arbitrary category $\mathcal{D}$. For an $n$-tuple of objects $\mathbf{X}=\left(X_{1}, \ldots, X_{n}\right)$ in $\mathcal{C}_{f}$, the $n$-cube $H_{B}^{\mathbf{X}}$ in $\mathcal{D}$ is defined by

$$
H_{B}^{\mathbf{X}}(S)=H\left(X_{B}^{1}(S), \ldots, X_{B}^{n}(S)\right),
$$

where

$$
X_{B}^{i}(S)= \begin{cases}X_{i} & \text { if } i \notin S, \\ B & \text { if } i \in S\end{cases}
$$

The image of the inclusion map $S \subset T$ under $H_{B}^{\mathrm{X}}$ is induced by the maps $\beta_{X_{i}}$.

We will make use of an $n$-cube of this type obtained by using the functor $\sqcup^{n}$ : $\mathcal{C}_{f}^{\times n} \rightarrow \mathcal{C}_{f}$ with

$$
\sqcup^{n}\left(X_{1}, \ldots, X_{n}\right)=X_{1} \coprod_{A} \cdots \coprod_{A} X_{n} .
$$

In particular, the 2-cube $\left(\sqcup^{2}\right)_{B}^{(X, Y)}$ is the square diagram

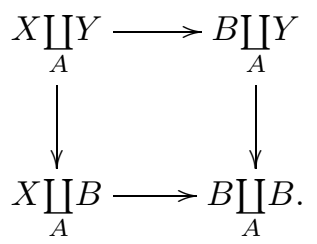

We will also make use of an $n$-cubical diagram that exploits the fact that every object in $\mathcal{C}_{f}$ is equipped with a map from $A$. Recall that we assume that the map $A \rightarrow X$ is a cofibration.

Example 2.9. For an object $X$ in $\mathcal{C}_{f}$, let $\alpha_{X}: A \rightarrow X$ denote the cofibration from the initial object to $X$. Let $H: \mathcal{C}_{f}^{\times n} \rightarrow \mathcal{D}$ be a functor of $n$ variables from $\mathcal{C}_{f}$ to an arbitrary category $\mathcal{D}$. Then for an $n$-tuple of objects $\mathbf{X}=\left(X_{1}, \ldots, X_{n}\right)$ in $\mathcal{C}_{f}$, the $n$-cube $H_{\mathbf{X}}^{A}: P(\mathbf{n}) \rightarrow \mathcal{D}$ is defined by

$$
H_{\mathbf{X}}^{A}(S)=H\left(X_{1}^{A}(S), \ldots, X_{n}^{A}(S)\right),
$$

where

$$
X_{i}^{A}(S)= \begin{cases}A & \text { if } i \notin S, \\ X_{i} & \text { if } i \in S .\end{cases}
$$

The image of the inclusion map $S \subset T$ under $H_{\mathbf{X}}^{A}$ is induced by the maps $\alpha_{X_{i}}$.

Again, the functor $\sqcup^{n}$ produces useful examples. The 2 -cube $\left(\sqcup^{2}\right)_{(X, Y)}^{A}$ is the square diagram

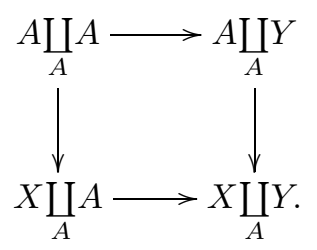

Since $A \coprod_{A} A=A, A \coprod_{A} Y=Y$ and $X \coprod_{A} A=X$, the diagram is a diagram which defines the coproduct in $\mathcal{C}_{f}$. In particular, it is homotopy cocartesian, as defined below. 
More generally, we are interested in $n$-cubes that are pullbacks or pushouts up to homotopy. In particular, we use the notions of homotopy cartesian and cocartesian diagrams introduced in [9]. To define these terms, we let $\mathcal{P}_{0}(\mathbf{n})$ be the full subcategory of $\mathcal{P}(\mathbf{n})$ determined by the non-empty subsets of $\mathbf{n}$ and $\mathcal{P}_{1}(\mathbf{n})$ be the full subcategory of $\mathcal{P}(\mathbf{n})$ determined by the subsets other than $\mathbf{n}$ itself.

Definition 2.10. Let $\chi$ be an $n$-cubical diagram in a model category $\mathcal{D}$.

- There are natural maps from the initial vertex $\chi(\emptyset)$ to $\operatorname{holim}_{S \in \mathcal{P}_{0}(\mathbf{n})} \chi(S)$ and $\operatorname{hocolim}_{S \in \mathcal{P}_{1}(\mathbf{n})} \chi(S)$ to the terminal vertex $\chi(\mathbf{n})$ determined by the compositions

$$
\chi(\emptyset)=\lim (\chi) \rightarrow \operatorname{holim}_{\mathcal{P}(\mathbf{n})} \chi \rightarrow \operatorname{holim}_{\mathcal{P}_{0}(\mathbf{n})} \chi,
$$

and

$$
\operatorname{hocolim}_{\mathcal{P}_{1}(\mathbf{n})} \chi \rightarrow \operatorname{hocolim}_{\mathcal{P}(\mathbf{n})} \chi \rightarrow \operatorname{colim}_{\mathcal{P}(\mathbf{n})} \chi=\chi(\mathbf{n}),
$$
respectively.

- We say that $\chi$ is homotopy cartesian if the map from the initial vertex $\chi(\emptyset)$ to holim $\operatorname{S\in \mathcal {P}}_{0}(\mathbf{n}) \chi(S)$ is a weak equivalence.

- We say that $\chi$ is homotopy cocartesian if the map from hocolim $_{S \in \mathcal{P}_{1}(\mathbf{n})} \chi(S)$ to the terminal vertex $\chi(\mathbf{n})$ is a weak equivalence.

- We say that $\chi$ is strongly homotopy cocartesian if each of its 2 -faces is homotopy cocartesian.

Following 9] we generally omit the term "homotopy" when speaking of these types of diagrams.

\section{Cross EFFECTS}

The cross effects for functors of abelian categories were introduced by Eilenberg and Mac Lane in [5]. Given a functor $F$ between two abelian categories and a positive integer $n$, Eilenberg and Mac Lane defined a functor of $n$ variables, $c r_{n} F$, that measures in some sense the extent to which $F$ fails to be additive. Drawing on their ideas, the second and third authors of this paper used the cross effects to define the degree of a functor and construct degree $n$ polynomial approximations to functors from a pointed category to an abelian category in 13 . The construction of the polynomial approximations depended on showing that the cross effect functors were parts of adjoint pairs and as such could be used to produce cotriples and cotriple resolutions that readily yielded the desired approximations.

In the present work, we extend these ideas to functors whose domain category is not pointed and whose target is not necessarily abelian. While some of the results of 13 carry through to this new context quite easily, others do not. In particular, identifying the adjoint pair that yields the desired cotriple requires a different approach. We use this section to adapt cross effects and the notion of the degree of a functor to a setting where the domain is of the form $\mathcal{C}_{f}$ that we introduced in section 2.1. We also identify adjoint pairs and cotriples associated to cross effects that we need.

Throughout this section we work with functors from the category $\mathcal{C}_{f}$ to the target category $\mathcal{D}$ where $\mathcal{C}_{f}$ and $\mathcal{D}$ are both simplicial model categories as described in section 2.1. In our constructions, we need to use the fact that the target category (but not the domain category) is pointed. Thus we assume that $\mathcal{D}$ is pointed and denote the initial/final object by $\star$. To ensure that our homotopy limit constructions 
behave nicely with respect to weak equivalences, we further assume that all functors take fibrant values in $\mathcal{D}$.

The cross effect functors will be functors of functors. For this reason, we will often need to consider the "category" of functors from one category to another. Strictly speaking, we cannot do so since these categories rarely have sets of morphisms (which are defined by natural transformations). In practice, this can often be resolved. The functors from $\mathcal{C}$ to $\mathcal{D}$ will form a category if $\mathcal{C}$ is skeletally-small or if we are careful to fix a suitable universe of sets in which to work (as in [10]). For the remainder of this paper, we assume that we are in a situation in which such categories of functors make sense. We use $\operatorname{Fun}\left(\mathcal{C}_{f}, \mathcal{D}\right)$ to denote the category of functors from $\mathcal{C}_{f}$ to $\mathcal{D}$ that preserve weak equivalences, and $\operatorname{Fun}\left(\mathcal{C}_{f}^{\times n}, \mathcal{D}\right)$ to denote the category of functors of $n$ variables from $\mathcal{C}_{f}$ to $\mathcal{D}$ that preserve weak equivalences.

3.1. Iterated fibers and cross effects. Our first step is to define cross effects for our context. The definition of the $n$th cross effect functor involves the iterated fibers of $n$-cubical diagrams associated with the $n$-fold coproduct functor $\sqcup^{n}$. To better understand the definition, consider a commuting square of objects in $\mathcal{D}$ :

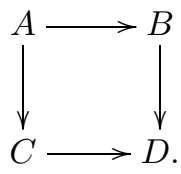

If we take homotopy fibers vertically, we obtain a map of homotopy fibers:

$$
\text { hofiber }\left(\begin{array}{c}
A \\
\downarrow \\
\\
C
\end{array}\right) \rightarrow \text { hofiber }\left(\begin{array}{c}
B \\
\downarrow \\
D
\end{array}\right) \text {. }
$$

We can take the homotopy fiber of this map to obtain an object, $X$, in $\mathcal{D}$, that we call the iterated fiber of the diagram:

$$
X=\text { hofiber }\left(\text { hofiber }\left(\begin{array}{c}
A \\
\downarrow \\
\\
C
\end{array}\right) \rightarrow \text { hofiber }\left(\begin{array}{c}
B \\
\downarrow \\
D
\end{array}\right)\right) .
$$

Recall that the homotopy fiber of a map such as $A \rightarrow C$ is defined to be the homotopy limit of the diagram

$$
A \rightarrow C \leftarrow \star
$$

Using this, we see that $X$ is the homotopy limit of

$$
\operatorname{holim}\left(\begin{array}{c}
A \\
\downarrow \\
C \\
\uparrow \\
\star
\end{array}\right) \rightarrow \operatorname{holim}\left(\begin{array}{c}
B \\
\downarrow \\
D \\
\uparrow \\
\uparrow \\
\star
\end{array}\right) \leftarrow \star
$$


Using properties (1) and (3) of Lemma 2.5] we see that $X$ is the homotopy limit of

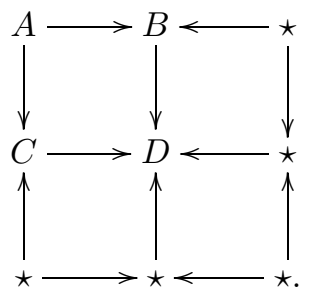

This is also isomorphic to

$$
\operatorname{hofiber}\left(\begin{array}{c}
\operatorname{hofiber}(A \rightarrow B) \\
\downarrow \\
\operatorname{hofiber}(C \rightarrow D)
\end{array}\right)
$$

and, as a consequence, we see that

$$
\operatorname{hofiber}\left(\begin{array}{c}
\operatorname{hofiber}(A \rightarrow B) \\
v_{\operatorname{hofiber}(C \rightarrow D)}
\end{array}\right) \cong \operatorname{hofiber}\left(\operatorname{hofiber}\left(\begin{array}{c}
A \\
\downarrow \\
\downarrow \\
C
\end{array}\right) \rightarrow \operatorname{hofiber}\left(\begin{array}{c}
B \\
\downarrow \\
\downarrow
\end{array}\right)\right) \text {. }
$$

In other words, the order of the directions in which we take fibers does not matter when defining the iterated fiber of $X$. We generalize this to define the iterated fiber of an $n$-cube. To do so we define a new diagram associated to an $n$-cubical diagram.

Definition 3.1. Let $\mathcal{X}$ be an $n$-cubical diagram in $\mathcal{D}$. The associated $\left(\mathcal{P}_{0}(\mathbf{2})\right)^{\times n_{-}}$ diagram $\mathcal{X}^{*}$ assigns to the $n$-tuple of sets $\left(S_{1}, \ldots, S_{n}\right)$ in $\mathcal{P}_{0}(\mathbf{2})$ the object

$$
\mathcal{X}^{*}\left(S_{1}, \ldots, S_{n}\right)= \begin{cases}\star & \text { if } S_{i}=\{2\} \text { for at least one } i, \\ \mathcal{X}\left(\left\{i \mid S_{i}=\{1,2\}\right\}\right) & \text { otherwise. }\end{cases}
$$

$\mathcal{X}^{*}$ takes a morphism of sets in $\left(\mathcal{P}_{0}(\mathbf{2})\right)^{\times n}, \phi:\left(S_{1}, \ldots, S_{n}\right) \rightarrow\left(T_{1}, \ldots, T_{n}\right)$ to the map $\mathcal{X}^{*}(\phi)$ defined as follows:

$$
\mathcal{X}^{*}(\phi)= \begin{cases}\mathcal{X}\left(\left\{i \mid S_{i}=\{1,2\}\right\} \subseteq\left\{j \mid T_{j}=\{1,2\}\right\}\right), & S_{i}, T_{j} \neq\{2\}, 1 \leq i, j \leq n, \\ \mathcal{X}^{*}\left(S_{1}, S_{2}, \ldots, S_{n}\right) \rightarrow \star, & T_{j}=\{2\} \text { for some } j, \\ \star \rightarrow \mathcal{X}^{*}\left(T_{1}, \ldots, T_{n}\right), & S_{i}=\{2\} \text { for some } i,\end{cases}
$$

where the second and third maps are uniquely determined by the fact that $\star$ is the initial/final object in $\mathcal{D}$.

We note that if $\mathcal{X}$ is the diagram in (2), then $\mathcal{X}^{*}$ is the diagram in (3).

Definition 3.2. Let $\mathcal{X}$ be an $n$-cubical diagram in $\mathcal{D}$. The iterated (homotopy) fiber of $\mathcal{X}$, denoted ifiber $\mathcal{X}$, is given by

$$
\text { ifiber } \mathcal{X}=\operatorname{holim}_{\left(\mathcal{P}_{0}(\mathbf{2})\right) \times n} \mathcal{X}^{*}
$$


As demonstrated in the case of a 2-cube, one can think of the iterated fiber of an $n$-cube as the object constructed by first taking fibers in one direction, $U \rightarrow U \cup\{i\}$, in the $n$-cube, then taking fibers of the resulting fibers in another direction, and continuing until one has exhausted all independent directions in the cube. As an immediate consequence we have the following.

Lemma 3.3. Let $\mathcal{X}$ be an $n$-cube and let $\mathcal{X}_{1}$ and $\mathcal{X}_{2}$ be $(n-1)$-cubes with $\mathcal{X}=$ $\mathcal{X}_{1} \rightarrow \mathcal{X}_{2}$. Then

$$
\text { ifiber } \mathcal{X} \cong \text { hofiber }\left(\operatorname{ifiber}\left(\mathcal{X}_{1}\right) \rightarrow \operatorname{ifiber}\left(\mathcal{X}_{2}\right)\right) \text {. }
$$

We use iterated fibers to define the $n$th cross effect of a functor.

Definition 3.4. Let $F: \mathcal{C}_{f} \rightarrow \mathcal{D}$ be a functor. The $n$th cross effect of $F$ is the functor of $n$ variables $c r_{n} F: \mathcal{C}_{f}^{\times n} \rightarrow \mathcal{D}$ that for an $n$-tuple $\mathbf{X}=\left(X_{1}, X_{2}, \ldots, X_{n}\right)$ is the iterated fiber of the $n$-cube $F\left(\left(\sqcup^{n}\right)_{B}^{\mathbf{X}}\right)$ that results from applying the functor $F$ to the $n$-cubical diagram $\left(\sqcup^{n}\right)_{B}^{\mathbf{X}}$ (as defined in Example2.8). When we precompose $c r_{n}$ with the diagonal functor $\Delta: X \mapsto(X, X, \ldots, X)$, the result is a functor from $\mathcal{C}_{f}$ to $\mathcal{D}$. We use $\perp_{n}$ to denote this composition, that is, $\perp_{n} F(X)=c r_{n} F(X, X, \ldots, X)$.

When $\mathcal{C}_{f}$ is pointed and $\mathcal{D}$ is abelian, these definitions agree with those of [13.

Example 3.5. For $n=2, c r_{2} F\left(X_{1}, X_{2}\right)$ is the iterated fiber of

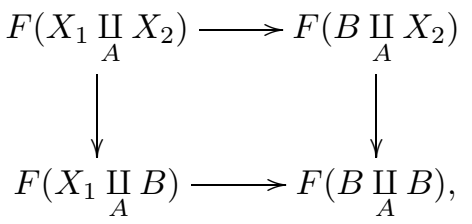

and $\perp_{2} F(X)$ is the iterated fiber of

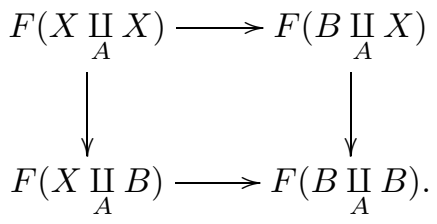

As discussed at the beginning of this section, the cross effect functors play an essential role in the construction of Taylor towers in [13. When $\mathcal{C}$ is a pointed category and $\mathcal{A}$ is an abelian category, it is straightforward to show that $\left(\Delta^{*}, c r_{n}\right)$ is an adjoint pair of functors. (Here $\Delta^{*}$ denotes precomposition with the diagonal functor.) As a consequence, $\perp_{n}=\Delta^{*} \circ \mathrm{cr}_{n}$ is a cotriple - this was used to construct the $(n-1)$ st term in the Taylor tower of a functor $F$.

In trying to replicate this process for functors from $\mathcal{C}_{f}$ to $\mathcal{D}$, one encounters an obstruction almost immediately. The functors $\Delta^{*}$ and $c r_{n}$ no longer form a strict adjoint pair. Goodwillie has shown that they form an adjoint pair up to weak equivalence in a topological setting [10, but something more is needed to show that $\perp_{n}$ is a cotriple. Our solution is to recognize that $\perp_{n}$ arises naturally from a pair of adjunctions. The first involves the functor $t$ defined as follows. 
Definition 3.6. For a functor $H: \mathcal{C}_{f}^{\times n} \rightarrow \mathcal{D}$, the functor $t H: \mathcal{C}_{f}^{\times n} \rightarrow \mathcal{D}$ is defined for an $n$-tuple $\mathbf{X}=\left(X_{1}, X_{2}, \ldots, X_{n}\right)$ of objects in $\mathcal{C}_{f}$ by

$$
t H\left(X_{1}, \ldots, X_{n}\right)=\operatorname{ifiber}\left(H_{B}^{\mathbf{X}}\right) .
$$

This is the reduction of $H$ in each variable in the sense of Goodwillie.

Remark 3.7. Note that for $H=\sqcup^{n}$, the $n$-fold coproduct functor, a functor $F$ : $\mathcal{C}_{f} \rightarrow \mathcal{D}$ and an $n$-tuple $\left(X_{1}, \ldots, X_{n}\right)$ of objects in $\mathcal{C}_{f}$,

$$
c r_{n} F\left(X_{1}, \ldots, X_{n}\right)=t\left(F \circ \sqcup^{n}\right)\left(X_{1}, \ldots, X_{n}\right) .
$$

In the next two subsections, we show that $t$ is a cotriple on $\operatorname{Fun}\left(\mathcal{C}_{f}^{\times n}, \mathcal{D}\right)$ and identify an associated adjunction. The other adjoint pair involves $\sqcup^{n}$ and the diagonal functor.

3.2. $t$ is a cotriple. Recall that a cotriple on a category $\mathcal{A}$ consists of a functor $\perp: \mathcal{A} \rightarrow \mathcal{A}$ together with natural transformations $\epsilon: \perp \rightarrow \mathrm{id}_{\mathcal{A}}$ and $\delta: \perp \rightarrow \perp \perp$ such that the following diagrams commute:
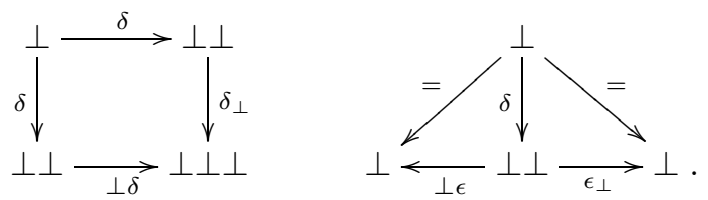

Our first goal is to prove the following.

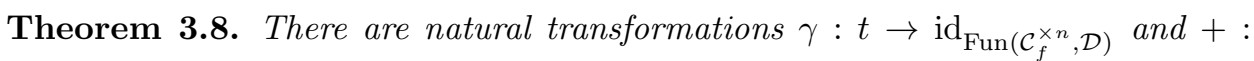
$t \rightarrow t t$ such that $(t,+, \gamma)$ is a cotriple on $\operatorname{Fun}\left(\mathcal{C}_{f}^{\times n}, \mathcal{D}\right)$.

To prove this theorem we begin by defining the natural transformations $\gamma$ : $t \rightarrow \operatorname{id}_{\operatorname{Fun}\left(\mathcal{C}_{f}^{\times n}, \mathcal{D}\right)}$ and $+: t \rightarrow t t$. Both of these natural transformations will be determined by applying property (2) of Lemma 2.5 to maps of the indexing sets used in the homotopy inverse limits that define $t H$ and $t t H$. We begin with $\gamma$.

Definition 3.9. Let $H: \mathcal{C}_{f}^{\times n} \rightarrow \mathcal{D}$ and $\mathbf{X}=\left(X_{1}, \ldots, X_{n}\right)$ be an object in $\mathcal{C}_{f}^{\times n}$. Consider the inclusion, $g:\{(\{1\},\{1\}, \ldots,\{1\})\} \rightarrow\left(\mathcal{P}_{0}(\mathbf{2})\right)^{\times n}$. By properties $(2)$ and (4) of Lemma 2.5, this induces a natural transformation

$$
\operatorname{holim}_{\left(\mathcal{P}_{0}(\mathbf{2})\right)^{\times n}}\left(H_{B}^{\mathbf{X}}\right)^{*} \longrightarrow \underset{\{(\{1\},\{1\}, \ldots,\{1\})\}}{\operatorname{holim}}\left(\left(H_{B}^{\mathbf{X}}\right)^{*} \circ g\right) \cong H\left(X_{1}, \ldots, X_{n}\right),
$$

which is natural in both $H$ and $\mathbf{X}$. This gives us the natural transformation $\gamma$ : $t \rightarrow \operatorname{id}_{\operatorname{Fun}\left(\mathcal{C}_{f}^{\times n}, \mathcal{D}\right)}$.

The definition of the second natural transformation requires understanding the two-fold iteration of $t$ as the iterated fiber of a single cube. Note that there is an isomorphism $\mathcal{P}(\mathbf{n}) \times \mathcal{P}(\mathbf{n}) \cong \mathcal{P}(\mathbf{2 n})$ realized for example by sending $(S, T) \in$ $\mathcal{P}(\{1,2, \ldots, n\}) \times \mathcal{P}(\{n+1, \ldots, 2 n\})$ to $S \cup T$. Given this, we can treat $2 n$-cubes as $(\mathcal{P}(\mathbf{n}) \times \mathcal{P}(\mathbf{n}))$-diagrams.

Lemma 3.10. Let $H: \mathcal{C}_{f}^{\times n} \rightarrow \mathcal{D}$ and $\mathbf{X}=\left(X_{1}, X_{2}, \ldots, X_{n}\right)$ be an object in $\mathcal{C}_{f}^{\times n}$. Then $\operatorname{tt} H\left(X_{1}, X_{2}, \ldots, X_{n}\right)$ is isomorphic to the iterated fiber of the $\mathcal{P}(\mathbf{n}) \times \mathcal{P}(\mathbf{n})$ diagram defined by

$$
(S, T) \in \mathcal{P}(\mathbf{n}) \times \mathcal{P}(\mathbf{n}) \mapsto H_{B}^{\mathbf{X}}(S \cup T) .
$$


Proof. By definition, $t t H\left(X_{1}, \ldots, X_{n}\right)$ is the iterated fiber of the $n$-cubical diagram

$$
S \mapsto(t H)_{B}^{\mathbf{X}}(S)
$$

But for each $S \subseteq \mathbf{n},(t H)_{B}^{\mathbf{X}}(S)$ is itself the iterated fiber of the $n$-cubical diagram

$$
T \mapsto(H)_{B}^{\mathbf{X}(S)}(T),
$$

where $\mathbf{X}(S)=\left(X^{1}(S), \ldots, X^{n}(S)\right)$ is as defined in Example 2.8. As observed in the discussion preceding Definition 3.1, homotopy fibers commute isomorphically in $\mathcal{D}$. From this we see that $t t H(\mathbf{X})$ is the iterated fiber of the $\mathcal{P}(\mathbf{n}) \times \mathcal{P}(\mathbf{n})$-diagram

$$
(S, T) \mapsto H_{B}^{\mathbf{X}(S)}(T) .
$$

It is easy to check that $H_{B}^{\mathbf{X}(S)}(T)=H_{B}^{\mathbf{X}}(S \cup T)$, and the result follows from this.

Remark 3.11. In some instances, it will be more convenient to treat the $\mathcal{P}(\mathbf{n}) \times \mathcal{P}(\mathbf{n})$ diagram $(S, T) \in \mathcal{P}(\mathbf{n}) \times \mathcal{P}(\mathbf{n}) \mapsto H_{B}^{\mathbf{X}}(S \cup T)$ of the previous lemma as the $\mathbf{2 n}$-cube $\widetilde{H}_{B}^{\mathrm{X}}$ given by

$$
U \subseteq \mathbf{2 n} \mapsto H\left(M_{1}(U), M_{2}(U), \ldots, M_{n}(U)\right)
$$

where

$$
M_{i}(U)= \begin{cases}X_{i} & \text { if }\{i, n+i\} \cap U=\emptyset, \\ B & \text { if }\{i, n+i\} \cap U \neq \emptyset .\end{cases}
$$

Definition 3.12. Let $+:\left(\mathcal{P}_{0}(\mathbf{2})\right)^{\times 2 n} \rightarrow\left(\mathcal{P}_{0}(\mathbf{2})\right)^{\times n}$ be the map

$$
\left(S_{1}, S_{2}, \ldots, S_{n}, T_{1}, \ldots, T_{n}\right) \mapsto\left(V_{1}, V_{2}, \ldots, V_{n}\right) \text {, }
$$

where

$$
V_{i}= \begin{cases}S_{i} \cup T_{i} & \text { if } S_{i}, T_{i} \neq\{2\}, \\ \{2\} & \text { if } S_{i}=\{2\} \text { or } T_{i}=\{2\} .\end{cases}
$$

By Lemma 2.5 (2), the set map $+:\left(\mathcal{P}_{0}(\mathbf{2})\right)^{\times 2 n} \rightarrow\left(\mathcal{P}_{0}(\mathbf{2})\right)^{\times n}$ induces a map

$$
t H(\mathbf{X})=\operatorname{holim}_{\left(\mathcal{P}_{0}(\mathbf{2})\right)^{\times n}}\left(H_{B}^{\mathbf{X}}\right)^{*} \rightarrow \operatorname{holim}_{\left(\mathcal{P}_{0}(\mathbf{2})\right)^{\times 2 n}}\left(\left(H_{B}^{\mathbf{X}}\right)^{*} \circ+\right) .
$$

But, it is straightforward to show that $\left(\widetilde{H}_{B}^{\mathbf{X}}\right)^{*}=\left(H_{B}^{\mathbf{X}}\right)^{*} \circ+$. Hence, (4) gives us a natural transformation

$$
+_{H}: t H \rightarrow t t H .
$$

With these definitions, we prove Theorem 3.8

Proof. We begin by showing that the diagram

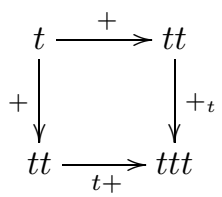

commutes. Let $H: \mathcal{C}_{f}^{\times n} \rightarrow \mathcal{D}$ and $\mathbf{X}=\left(X_{1}, \ldots, X_{n}\right)$ be an $n$-tuple of objects in $\mathcal{C}_{f}$. Note that, as is the case with $t t H(\mathbf{X}), \operatorname{ttt} H(\mathbf{X})$ can be realized as the iterated fiber of a $\mathbf{3 n}$-cube. In particular, $t t t H(\mathbf{X})$ is the iterated fiber of the $\mathbf{3 n}$-cube that assigns to the set $U \subseteq \mathbf{3 n}$ the object

$$
H\left(J_{1}(U), \ldots, J_{n}(U)\right)
$$


with

$$
J_{i}(U)= \begin{cases}X_{i} & \text { if }\{i, n+i, 2 n+i\} \cap U=\emptyset, \\ B & \text { otherwise. }\end{cases}
$$

Hence, $\operatorname{tttH}(\mathbf{X})$ can be treated as the holim of a $\left(\mathcal{P}_{0}(\mathbf{2})\right)^{\times 3 n}$-diagram. From this point of view, we see that (6) commutes by noting that the maps are induced by the commuting diagram of set maps

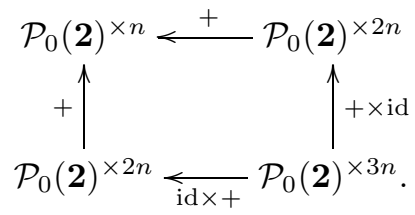

To see that

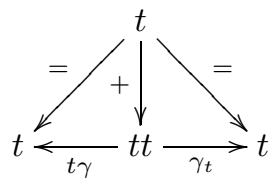

commutes, we note that $t \gamma$ and $\gamma_{t}$ are induced at the indexing set level by the maps

$$
\iota_{1}:\left(\mathcal{P}_{0}(\mathbf{2})\right)^{\times n} \rightarrow\left(\mathcal{P}_{0}(\mathbf{2})\right)^{\times 2 n}, \iota_{1}:\left(S_{1}, \ldots, S_{n}\right) \mapsto\left(S_{1}, \ldots, S_{n},\{1\}, \ldots,\{1\}\right),
$$

and

$$
\iota_{2}:\left(\mathcal{P}_{0}(\mathbf{2})\right)^{\times n} \rightarrow\left(\mathcal{P}_{0}(\mathbf{2})\right)^{\times 2 n}, \iota_{2}:\left(S_{1}, \ldots, S_{n}\right) \mapsto\left(\{1\}, \ldots,\{1\}, S_{1}, \ldots, S_{n}\right),
$$

respectively. It is straightforward to check that $+\circ \iota_{1}$ and $+\circ \iota_{2}$ are the identity maps on $\left(\mathcal{P}_{0}(\mathbf{2})\right)^{\times n}$. From this it follows that $t \gamma \circ+$ and $\gamma_{t} \circ+$ are the identity on $t$.

3.3. $\perp_{n}$ is a cotriple. To establish that $\perp_{n}$ is a cotriple, we describe an adjunction determined by the cotriple $t$. Categories equipped with cotriples have a related category of coalgebras, related to the original category by a forgetful-cofree adjunction.

Definition 3.13 (15, Definition VI.2, dualized]). If $(\perp, \delta, \epsilon)$ is a cotriple on a category $\mathcal{B}$, then the category $\mathcal{B}_{\perp}$ of $\perp$-coalgebras is the category whose objects are pairs $(B, \beta)$, where $B \in O b(\mathcal{B})$ and $\beta: B \rightarrow \perp B$, which satisfy

$$
\perp \beta \circ \beta=\delta_{B} \circ \beta \text { and } \epsilon_{B} \circ \beta=I d_{B} .
$$

A morphism $f:(B, \beta) \rightarrow\left(B^{\prime}, \beta^{\prime}\right)$ in $\mathcal{B}_{\perp}$ is a morphism $f: B \rightarrow B^{\prime}$ in $\mathcal{B}$ such that $\perp f \circ \beta=\beta^{\prime} \circ f$.

Thus, the category of $t$-coalgebras, $\operatorname{Fun}\left(\mathcal{C}_{f}^{\times n}, \mathcal{D}\right)_{t}$, consists of functors $G: \mathcal{C}_{f}^{\times n} \rightarrow$ $\mathcal{D}$ that are equipped with a section $\beta: G \rightarrow t G$ to the natural transformation $\gamma_{G}: t G \rightarrow G$ which also makes the diagram

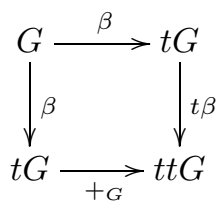


commute. For example, for any functor $G \in \operatorname{Fun}\left(\mathcal{C}_{f}^{\times n}, \mathcal{D}\right)$, there is an associated $t$-coalgebra $\left(t G,+_{G}\right)$. Let $t^{+}: \operatorname{Fun}\left(\mathcal{C}_{f}^{\times n}, \mathcal{D}\right) \rightarrow \operatorname{Fun}\left(\mathcal{C}_{f}^{\times n}, \mathcal{D}\right)_{t}$ be the free coalgebra functor, which is defined on objects by $t^{+}(G)=\left(t G,+_{G}\right)$.

Theorem 3.14. The functors

$$
\operatorname{Fun}\left(\mathcal{C}_{f}^{\times n}, \mathcal{D}\right) \underset{U^{+}}{\stackrel{t^{+}}{\rightleftarrows}} \operatorname{Fun}\left(\mathcal{C}_{f}^{\times n}, \mathcal{D}\right)_{t}
$$

are an adjoint pair of functors, with the forgetful functor $U^{+}$being the left adjoint.

Proof. The proof follows immediately from Theorem 3.8, since $(t,+, \gamma)$ forms a cotriple. The adjunction in question is the forgetful-cofree adjunction which exists for any category of coalgebras over a cotriple. The proof of this fact is formally dual to the proof of Theorem VI.2.1 found in [15] for algebras over triples.

We now turn our attention to the second adjoint pair of functors that we use to establish that $\perp_{n}$ is a cotriple.

Definition 3.15. Let

$$
\Delta^{*}: \operatorname{Fun}\left(\mathcal{C}_{f}^{\times n}, \mathcal{D}\right) \rightarrow \operatorname{Fun}\left(\mathcal{C}_{f}, \mathcal{D}\right)
$$

be the functor defined for a functor $H: \mathcal{C}_{f}^{\times n} \rightarrow \mathcal{D}$ by $\Delta^{*} H(X)=H(X, \ldots, X)$. Let

$$
\sqcup_{n}: \operatorname{Fun}\left(\mathcal{C}_{f}, \mathcal{D}\right) \rightarrow \operatorname{Fun}\left(\mathcal{C}_{f}^{\times n}, \mathcal{D}\right)
$$

be the functor defined by precomposition with the functor $\sqcup^{n}$ of Examples 2.8 and 2.9. That is, for a functor $F$

$$
\sqcup_{n}(F)\left(X_{1}, \ldots, X_{n}\right)=\left(F \circ \sqcup^{n}\right)\left(X_{1}, \ldots, X_{n}\right)=F\left(X_{1} \amalg_{A} \ldots \amalg_{A} X_{n}\right) .
$$

Proposition 3.16. The functors $\Delta^{*}$ and $\sqcup_{n}$ are an adjoint pair of functors, with $\Delta^{*}$ being the left adjoint.

Proof. Let $H: \mathcal{C}_{f}^{\times n} \rightarrow \mathcal{D}$ and $F: \mathcal{C}_{f} \rightarrow \mathcal{D}$. We must prove that there are isomorphisms

$$
\operatorname{hom}_{\mathrm{Fun}\left(\mathcal{C}_{f}, \mathcal{D}\right)}\left(\Delta^{*} H, F\right) \underset{\Psi}{\stackrel{\Phi}{\longleftarrow}} \operatorname{hom}_{\mathrm{Fun}\left(\mathcal{C}_{f}^{\times n}, \mathcal{D}\right)}\left(H, \sqcup_{n} F\right) .
$$

We do so by taking advantage of some coproduct properties.

In $\mathcal{C}_{f}$, the coproduct of $X$ and $Y$ is the pushout of

$$
Y \longleftarrow A \longrightarrow X
$$

which we denote $X \coprod_{A} Y$. When $X=Y$, the fact that $X \coprod_{A} X$ is a pushout means that we have a fold map $+: X \coprod_{A} X \rightarrow X$ that serves as a section to the inclusion $X \rightarrow X \coprod_{A} X$ into either term of the coproduct. Iterating this gives a fold map $+: \coprod_{n} X \rightarrow X$ that for each $1 \leq k \leq n$ is a section to $\iota_{k}: X \rightarrow \coprod_{n} X$, inclusion into the $k$ th term in the coproduct.

The map $\Phi$ sends a natural transformation $\sigma: \Delta^{*} H \rightarrow F$ to the natural transformation $\Phi \sigma:=\sqcup_{n} \sigma \circ H\left(i_{1}, \ldots, i_{n}\right)$. On the other hand, a natural transformation $\tau: H \rightarrow \sqcup_{n} F$ is sent to $\Psi \tau:=F(+) \circ \tau$. 
To see that $\Psi \Phi \sigma$ is equal to $\sigma$, consider the diagram:

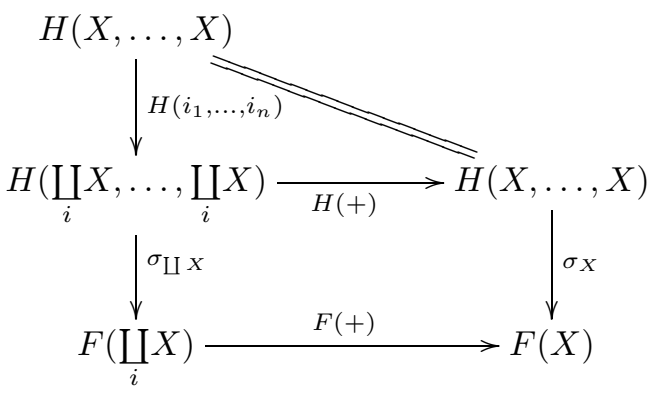

The bottom rectangle commutes by the naturality of $\sigma$, and the top triangle commutes because + is a section to each $i_{k}$. Going along the bottom and left edges of the diagram gives $\Psi \Phi \sigma$, while the right hand edge is just $\sigma$.

On the other hand, the diagram

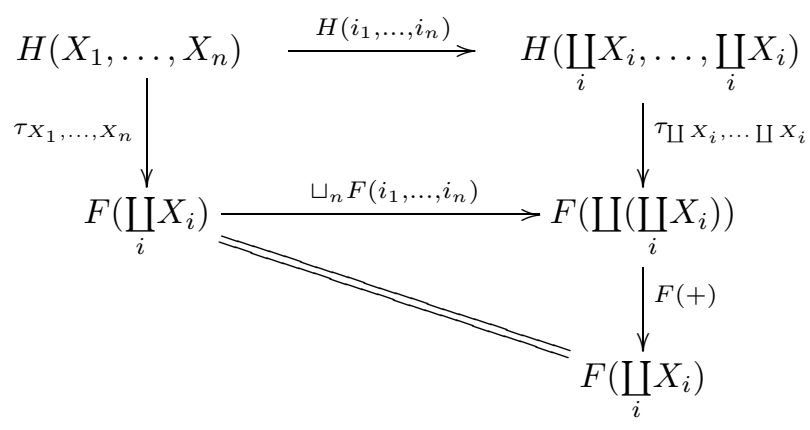

commutes for the same reasons as the previous diagram, and shows that $\Phi \Psi \tau=$ $\tau$.

Recall that for an adjoint pair of functors,

$$
F: \mathcal{C} \rightleftarrows \mathcal{D}: G
$$

where $G$ is the right adjoint, the composition $F \circ G$ forms a cotriple on $\mathcal{A}$. (See, for example, Appendix A.6 of [22].) Since $\perp_{n}$ is the composition of the left adjoint $\Delta^{*} \circ U^{+}$with the right adjoint $t^{+} \circ \sqcup_{n}$, it forms part of a cotriple. In particular, the counit for the adjunction produced by the pair $\left(\Delta^{*} \circ U^{+}, t^{+} \circ \sqcup_{n}\right)$ yields a natural transformation $\epsilon: \perp_{n} \rightarrow$ id. Also, a natural transformation $\delta: \perp_{n} \rightarrow \perp_{n} \perp_{n}$ is defined by $\Delta^{*} \circ U^{+}\left(\eta_{t^{+} \circ \sqcup_{n}}\right)$, where $\eta$ is a unit for the adjunction. This gives us the following.

Theorem 3.17. The functor and natural transformations

$$
\left(\perp_{n}, \delta: \perp_{n} \rightarrow \perp_{n} \perp_{n}, \epsilon: \perp_{n} \rightarrow \mathrm{id}\right)
$$

form a cotriple on the category of functors $\operatorname{Fun}\left(\mathcal{C}_{f}, \mathcal{D}\right)$.

3.4. Weakly reduced and degree $n$ functors. We finish this section by introducing some properties of functors that are related to $t$ and $c r_{n}$. 
Definition 3.18. A functor $F \in \operatorname{Fun}\left(\mathcal{C}_{f}^{\times n}, \mathcal{D}\right)$ is weakly $n$-reduced provided that $F\left(X_{1}, \ldots, X_{n}\right) \simeq \star$ whenever any $X_{i}=B$.

Proposition 3.19. Let $H: \mathcal{C}_{f}^{\times n} \rightarrow \mathcal{D}$. The functor $t H$ is a weakly n-reduced functor.

Proof. We assume that $X_{n}=B$. The argument in other cases is similar. We describe $H_{B}^{\mathbf{X}}$ as a map of two $(n-1)$-cubes: $\operatorname{top}_{B}^{\mathbf{X}} \rightarrow \operatorname{bottom} H_{B}^{\mathbf{X}}$. For $S \in$ $\mathcal{P}(\mathbf{n}-\mathbf{1})$,

$$
\operatorname{top} H_{B}^{\mathbf{X}}(S)=H_{B}^{\mathbf{X}}(S)
$$

and

$$
\operatorname{bottom} H_{B}^{\mathbf{X}}(S)=H_{B}^{\mathbf{X}}(S \cup\{n\}) .
$$

Consider the $(n-1)$-cube, $\partial H_{B}^{\mathbf{X}}$, obtained by taking the homotopy fiber of top $H_{B}^{\mathbf{X}} \rightarrow$ bottom $H_{B}^{\mathbf{X}}$. More explicitly, for $S \in P(\mathbf{n}-\mathbf{1})$,

$$
\partial H_{B}^{\mathbf{X}}(S)=\operatorname{hofib}\left(\operatorname{top} H_{B}^{\mathbf{X}}(S) \rightarrow \operatorname{bottom} H_{B}^{\mathbf{X}}(S)\right) .
$$

Using Lemma 3.3 one can show that ifiber $\left(\partial H_{B}^{\mathbf{X}}\right) \cong$ ifiber $\left(H_{B}^{\mathbf{X}}\right)$. For each $S \in$ $\mathcal{P}(\mathbf{n}-\mathbf{1})$, the map top $H_{B}^{\mathbf{X}}(S) \rightarrow$ bottom $H_{B}^{\mathbf{X}}(S)$ is the identity since $X_{n}=B$. Hence, $\partial H_{B}^{\mathbf{X}}(S) \simeq \star$ for each $S$, and so the iterated homotopy fiber of $\partial H_{B}^{\mathbf{X}}$ is equivalent to $\star$. As a consequence, the iterated homotopy fiber of $H_{B}^{\mathbf{X}}$ is as well.

Corollary 3.20. For a functor $F: \mathcal{C}_{f} \rightarrow \mathcal{D}, c r_{n} F$ is weakly n-reduced.

We use cross effects to define degree $n$ functors.

Definition 3.21. A functor $F: \mathcal{C}_{f} \rightarrow \mathcal{D}$ is degree $n$ if and only if for all $(n+1)$ tuples $\mathbf{X}$ of objects in $\mathcal{C}_{f}$,

$$
c r_{n+1} F(\mathbf{X}) \simeq \star
$$

Whenever a functor is degree $n$, it is also degree $m$ for any $m>n$. This is a consequence of the following lemma, which says that higher cross effects can be obtained by iterating second cross effects. The lemma implies, in particular, that if $c r_{n} F \simeq \star$, then $c r_{n+1} F \simeq \star$ as well.

Proposition 3.22. For a functor $F: \mathcal{C}_{f} \rightarrow \mathcal{D}$, and objects $X_{1}, \ldots, X_{n}, X_{n+1}$ in $\mathcal{C}_{f}, c r_{n} F\left(X_{1}, \ldots, X_{n-1},-\right)$ can be treated as a functor of one variable by holding the first $n-1$ variables fixed. The second cross effect of this functor is $\mathrm{cr}_{n+1}$. Specifically,

$$
c r_{2}\left[c r_{n} F\left(X_{1}, \ldots, X_{n-1},-\right)\right]\left(X_{n}, X_{n+1}\right) \simeq c r_{n+1} F\left(X_{1}, \ldots, X_{n+1}\right) .
$$

Proof. The proof makes repeated use of Lemma 3.3, which allows us to rewrite the diagrams whose iterated fibers yield $\operatorname{cr}_{2}\left(c r_{n} F\right)$ to obtain the $(n+1)$-cubical diagram defining $c r_{n+1}$. We begin by noting that

$$
c r_{2}\left[c r_{n} F\left(X_{1}, \ldots, X_{n-1},-\right)\right]\left(X_{n}, X_{n+1}\right)
$$


is defined to be the iterated homotopy fiber of the diagram

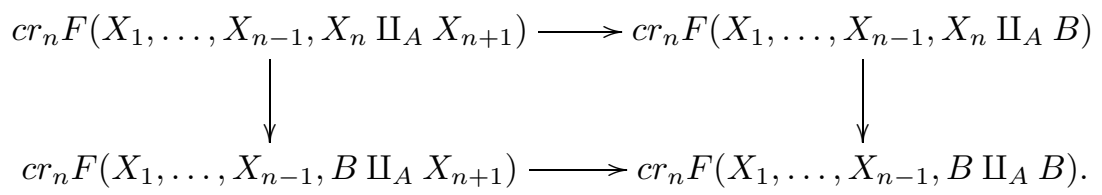

Each corner of this square diagram is the iterated homotopy fiber of an $n$-cube, so by Lemma 3.3 the iterated homotopy fiber of the diagram above can be written as the iterated homotopy fiber of the following 2 -cube of $n$-cubes:

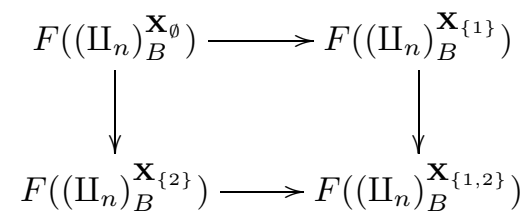

where

$$
\begin{aligned}
& \mathbf{X}_{\emptyset}=\left(X_{1}, \ldots, X_{n-1}, X_{n} \amalg_{A} X_{n+1}\right), \\
& \mathbf{X}_{\{1\}}=\left(X_{1}, \ldots, X_{n-1}, X_{n} \amalg_{A} B\right), \\
& \mathbf{X}_{\{2\}}=\left(X_{1}, \ldots, X_{n-1}, B \amalg_{A} X_{n+1}\right), \\
& \mathbf{X}_{\{1,2\}}=\left(X_{1}, \ldots, X_{n-1}, B \amalg_{A} B\right) .
\end{aligned}
$$

As in the proof of Proposition 3.19, we write each of the $n$-cubes in (7) as a map of $(n-1)$-cubes by replacing an $n$-cube $\chi$ with the map of $(n-1)$-cubes $\operatorname{top}(\chi) \rightarrow \operatorname{bottom}(\chi)$ where for $S \in P(\mathbf{n}-\mathbf{1})$,

$$
\begin{aligned}
& \operatorname{top}(\chi)(S)=\chi(S), \\
& \operatorname{bottom}(\chi)(S)=\chi(S \cup\{n\}) .
\end{aligned}
$$

By again applying Lemma [3.3, we can view the iterated fiber of (17) as the homotopy fiber of the map from the iterated homotopy fiber of the 2-cube of $(n-1)$ cubes

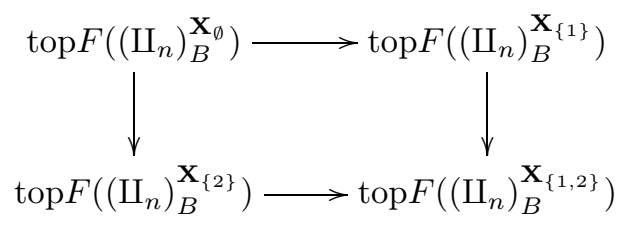

to the iterated homotopy fiber of

(bottom)

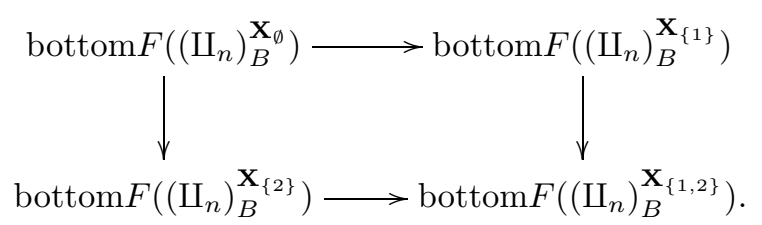

All four $(n-1)$-cubes in (bottom) are the same, so the iterated homotopy fiber of (bottom) is weakly equivalent to $\star$. Thus we can concentrate on determining the iterated homotopy fiber of (top). But this diagram can be rewritten as a single $(n+1)$-cube that is precisely the one whose iterated homotopy fiber is $c r_{n+1} F\left(X_{1}, \ldots, X_{n}, X_{n+1}\right)$. 


\section{DEGREE $n$ AND $n$-EXCISIVE FUNCTORS}

In the next section, we use the cotriple $\perp_{n+1}$ to construct a degree $n$ approximation to a functor $F$. In [10], Goodwillie shows how to construct an $n$-excisive approximation to a functor. We use this section to compare these two types of functors, showing that $n$-excisive functors are always degree $n$, and that degree $n$ functors behave like $n$-excisive functors on certain types of cubical diagrams (the condition we call $n$-excisive relative to $A$ ). We conclude by proving that the two notions are equivalent when $F$ is a functor that commutes with realization.

In this section, we work with functors $F: \mathcal{C}_{f} \rightarrow \mathcal{S}$, where $\mathcal{C}_{f}$ is the category of maps factoring $f: A \rightarrow B$ and $\mathcal{S}$ is a suitable model of spectra, such as in [6] or [12]. We let $\star$ be the initial/final object in $\mathcal{S}$. As in previous sections, we assume that $F$ preserves weak homotopy equivalences and takes values in fibrant objects. We also assume that $\mathcal{S}$ has functorial fibrant and cofibrant replacements. We first review the definition of $n$-excisive using the notions of cartesian and strongly cocartesian diagrams from Definition 2.10 .

Definition $4.1(9])$. A functor $F$ is $n$-excisive if and only if for every strongly cocartesian $(n+1)$-cube of objects in $\mathcal{C}_{f}, \chi$, applying $F$ yields a cartesian $(n+1)$ cube, $F(\chi)$.

For $\mathbf{X}=\left(X_{1}, \ldots, X_{n+1}\right)$ in $\mathcal{C}_{f}^{\times n+1}$, the cube $\left(\coprod_{n+1}\right)_{\mathbf{X}}^{A}$ of Example 2.9 is strongly homotopy cocartesian, recalling that each $A \rightarrow X_{i}$ is assumed to be a cofibration. In fact, any strongly homotopy cocartesian cube with initial vertex $A$ is weakly equivalent to one of this type by Proposition 2.2 of [9]. We prove that a degree $n$ functor will take strongly cocartesian diagrams like these to cartesian diagrams.

Definition 4.2. The functor $F: \mathcal{C}_{f} \rightarrow \mathcal{S}$ is $n$-excisive relative to $A$ if and only if $F\left(\left(\coprod_{n+1}\right)_{\mathbf{X}}^{A}\right)$ is cartesian for every $(n+1)$-tuple of objects $\mathbf{X}$ in $\mathcal{C}_{f}$.

Proposition 4.3. Let $F$ be a functor from $\mathcal{C}_{f}$ to $\mathcal{S}$. Let $n \geq 2$ be an integer. The functor $F$ is degree $n-1$ if and only if $F$ is $(n-1)$-excisive relative to $A$.

An integral part of the proof of this proposition will be the $n$-cube of $n$-cubes defined below.

Definition 4.4. Let $\mathbf{X}=\left(X_{1}, \ldots, X_{n}\right)$ be an $n$-tuple of objects in $\mathcal{C}_{f}$. Each of these objects is equipped with morphisms $\alpha_{X_{i}}: A \rightarrow X_{i}$ and $\beta_{X_{i}}: X_{i} \rightarrow B$ whose composition is $f$. We use $\widetilde{\mathbf{X}}: \mathcal{P}(\mathbf{n}) \times \mathcal{P}(\mathbf{n}) \rightarrow \mathcal{C}_{f}^{\times n}$ to denote the $n$-cube of $n$-cubes that is defined as follows. For $(S, T) \in \mathcal{P}(\mathbf{n}) \times \mathcal{P}(\mathbf{n}), \widetilde{\mathbf{X}}(S, T)$ is the $n$-tuple whose $i$ th object is

$$
(\widetilde{\mathbf{X}}(S, T))_{i}=\left\{\begin{array}{l}
A, \quad \text { if } i \notin S \cup T ; \\
X_{i}, \quad \text { if } i \in S, i \notin T ; \\
B, \quad \text { if } i \in T .
\end{array}\right.
$$

For any $i \notin T$ the map $\widetilde{\mathbf{X}}(S, T) \rightarrow \widetilde{\mathbf{X}}(S, T \cup\{i\})$ is induced by the map $f$ if $i \notin S$, and otherwise is induced by the map $\beta_{X_{i}}$. For $i \notin S$ the map $\widetilde{\mathbf{X}}(S, T) \rightarrow \widetilde{\mathbf{X}}(S \cup\{i\}, T)$ is induced by the map $\alpha_{X_{i}}$ if $i \notin T$ and otherwise is the identity map. 
The target category for our functor $F$ is assumed to be stable. Some of the subsequent results in this section hold in a more general context, but for our current applications using $\mathcal{S}$ as the target category suffices. This enables us to make use of the following observations.

Remark 4.5. An $n$-cubical diagram in $\mathcal{S}$ is cocartesian if and only if it is cartesian.

Remark 4.6. In $\mathcal{S}$, finite homotopy inverse limits commute up to weak equivalence with homotopy colimits of countable filtered diagrams. Thus, combined with the remark above one sees that finite homotopy inverse limits commute with homotopy colimits over $\Delta^{o p}$, as these homotopy colimits can be written using the filtration by skeleta as a countable filtered homotopy colimit of finite homotopy colimits.

Remark 4.7. Let $\chi$ be an $n$-cubical diagram. The total homotopy fiber of $\chi$, denoted $\operatorname{tfiber}(\chi)$, is the homotopy fiber of the map

$$
\chi(\emptyset) \rightarrow \underset{\mathcal{P}_{0}(\mathbf{n})}{\operatorname{holim}}(\chi)
$$

For $n$-cubes $\chi$ in $\mathcal{S}$, the iterated fiber and homotopy fiber of $\chi$ are weakly equivalent. See section 1 of [9] for details.

The next lemma restates two propositions from [9]. The lemma makes use of the fact that a map of two $n$-cubes, $\chi_{1} \rightarrow \chi_{2}$, is an $(n+1)$-cube.

Lemma $4.8([9,1.6,1.7])$. For any map $\chi_{1} \rightarrow \chi_{2}$ of $n$-cubes of objects in $\mathcal{S}$ :

- The $n$-cube $\chi_{1}$ is cartesian if $\chi_{2}$ is cartesian and the $(n+1)$-cube $\chi_{1} \rightarrow \chi_{2}$ is cartesian.

- The $n$-cube $\chi_{2}$ is cartesian if $\chi_{1}$ is cartesian and the $(n+1)$-cube $\chi_{1} \rightarrow \chi_{2}$ is cartesian.

- The $(n+1)$-cube $\chi_{1} \rightarrow \chi_{2}$ is cartesian if the $n$-cubes $\chi_{1}$ and $\chi_{2}$ are cartesian.

The proof of Proposition 4.3 relies on analyzing $\widetilde{\mathbf{X}}$ from several different perspectives. We single out two of these perspectives in the next remark. Recall from Definition 3.15 that $\sqcup_{n}$ denotes precomposition with the $n$-fold coproduct functor.

Remark 4.9. Let $\mathbf{X}$ be an $n$-tuple of objects in $\mathcal{C}_{f}$ and $F: \mathcal{C}_{f} \rightarrow \mathcal{S}$ be a functor.

(1) Fixing $S \in \mathcal{P}(\mathbf{n})$ yields an $n$-cube, $\sqcup^{n} \widetilde{\mathbf{X}}(S,-)$. When $S=\mathbf{n}$, this $n$-cube is $\left(\sqcup^{n}\right)_{B}^{\mathbf{X}}$, and, as such, is precisely the type of $n$-cube used to show that a functor is degree $n-1$. In other words, $F$ is degree $n-1$ if and only if the $n$-cube $\sqcup_{n} F(\widetilde{\mathbf{X}}(\mathbf{n},-))$ is cartesian.

(2) Fixing $T \in \mathcal{P}(\mathbf{n})$ yields another $n$-cube, $\sqcup^{n} \widetilde{\mathbf{X}}(-, T)$. When $T=\emptyset$, this $n$-cube is $\left(\sqcup^{n}\right)_{\mathbf{X}}^{A}$, and, as such, is precisely the type of $n$-cube used to show that a functor is $(n-1)$-excisive relative to $A$. In other words, $F$ is $(n-1)$ excisive relative to $A$ if and only if $\sqcup_{n} F(\widetilde{\mathbf{X}}(-, \emptyset))$ is cartesian. 
For example, taking the first point of view when $n=2$, we have the 2-cube of 2-cubes $\sqcup_{n} F(\widetilde{\mathbf{X}}(S, T))$ whose outer square is indexed by the $S$ variable:

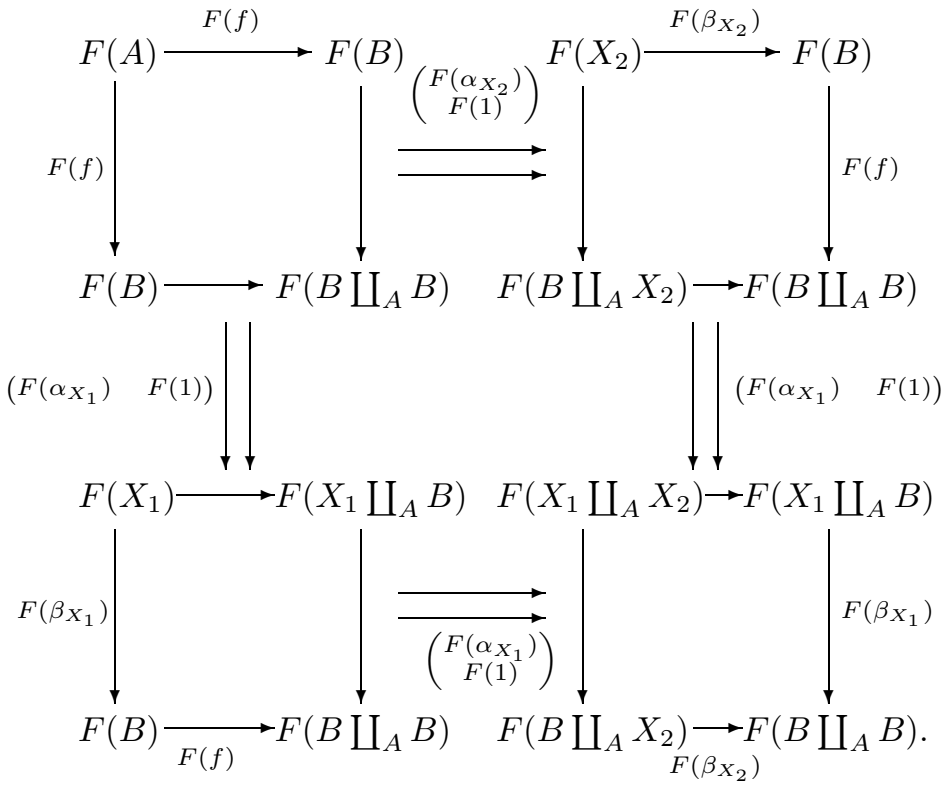

Taking the second point of view yields the 2-cube of 2-cubes $\sqcup_{2} F(\widetilde{\mathbf{X}}(S, T))$ whose outer square is indexed by the $T$ variable:

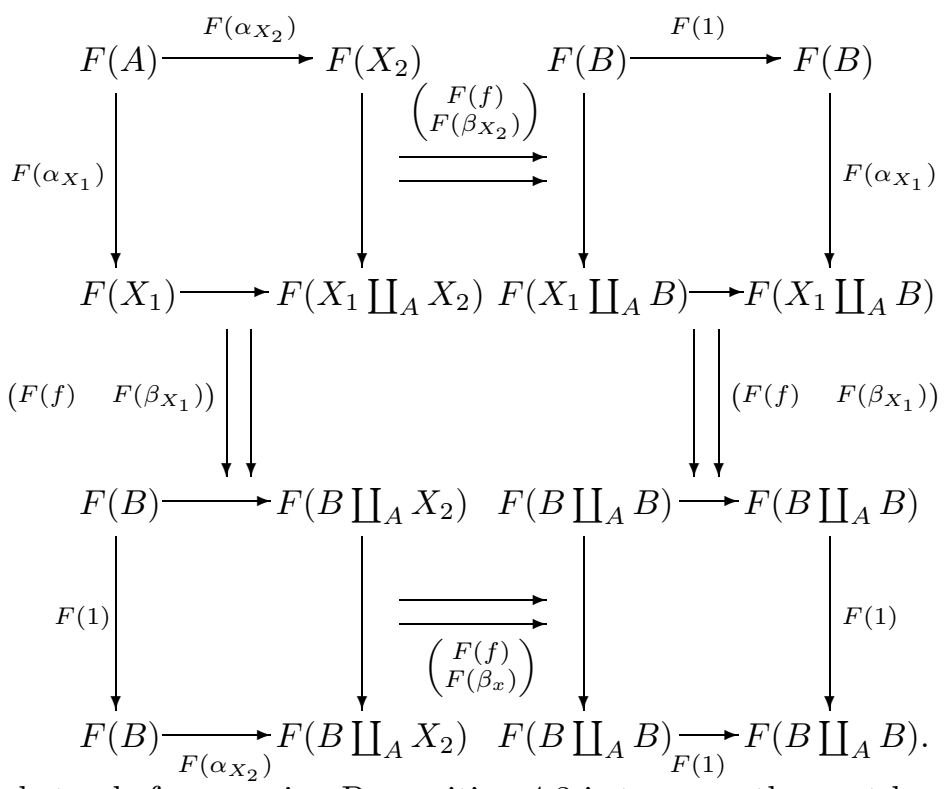

The final step before proving Proposition 4.3 is to prove the next lemma.

Lemma 4.10. Let $\mathbf{X}$ be an n-tuple of objects in $\mathcal{C}_{f}$ and $F: \mathcal{C}_{f} \rightarrow \mathcal{S}$ be a functor. If $F$ is $(n-1)$-excisive relative to $A$, then for each $S \subset \mathbf{n}, S \neq \mathbf{n}$, the $n$-cube $\sqcup_{n} F(\widetilde{\mathbf{X}}(S,-))$ is cartesian. 
Proof. We prove this by induction on the size of $S$. When $S=\emptyset, \sqcup^{n}(\widetilde{\mathbf{X}}(\emptyset,-))$ is the $n$-cube $\left(\sqcup^{n}\right)_{\mathbf{B}}^{A}$ where $\mathbf{B}=(B, B, \ldots, B)$. Since $F$ is $(n-1)$-excisive relative to $A$, we know that $\sqcup_{n} F(\widetilde{\mathbf{X}}(\emptyset,-))$ is cartesian.

Let $k<n$ and assume that $\sqcup_{n} F(\widetilde{\mathbf{X}}(R,-))$ is cartesian for all $R \subset \mathbf{n}$ with $|R|<k$. Let $S \subseteq \mathbf{n}$ with $|S|=k$. To establish that $\sqcup_{n} F(\widetilde{\mathbf{X}}(S,-))$ is cartesian, we will first show that the $S$-cube of $\mathbf{n}$-cubes

$$
\sqcup_{n} F(\widetilde{\mathbf{X}}(R, T)), \quad R \subseteq S, T \subseteq \mathbf{n},
$$

obtained by restricting $\widetilde{\mathbf{X}}$ to $\mathcal{P}(S) \times \mathcal{P}(\mathbf{n})$, is cartesian. To do so we view this $S$ cube of $\mathbf{n}$-cubes from a different perspective, in particular as an $S$-cube of $S$-cubes of $(\mathbf{n}-S)$-cubes.

Fix $R^{\prime} \subseteq S$ and consider the $S$-cube of $(\mathbf{n}-S)$-cubes, i.e., the $n$-cube, given by

$$
\sqcup_{n} F\left(\widetilde{\mathbf{X}}\left(R, R^{\prime} \cup U\right)\right),
$$

where $R$ varies over all subsets of $S$ and $U$ varies over all subsets of $\mathbf{n}-S$. We claim that for each $R^{\prime}$, this is a cartesian $n$-cube. When $R^{\prime}=\emptyset$, this follows because (9) is $F\left(\left(\sqcup^{n}\right)_{\mathbf{Y}}^{A}\right)$, where $\mathbf{Y}$ is the $n$-tuple whose $j$ th entry is

$$
(\mathbf{Y})_{j}= \begin{cases}X_{j} & \text { if } j \in S, \\ B & \text { if } j \notin S,\end{cases}
$$

and $F$ is $(n-1)$-excisive relative to $A$. Now suppose that $R^{\prime} \neq \emptyset$ and $i \in R^{\prime}$. In this case, we can view (9) as a map of $(|S|-1)$-cubes of $(\mathbf{n}-S)$-cubes:

$$
\sqcup_{n} F\left(\widetilde{\mathbf{X}}\left(R^{\prime \prime}, R^{\prime} \cup U\right)\right) \rightarrow \sqcup_{n} F\left(\widetilde{\mathbf{X}}\left(R^{\prime \prime} \cup\{i\}, R^{\prime} \cup U\right)\right),
$$

where $R^{\prime \prime} \subseteq S-\{i\}$ and $U \subseteq \mathbf{n}-S$ vary. By definition, the map in (10) is the identity for each choice of $R^{\prime \prime}$ and $U$. Applying Lemma 4.8, we see that the $S$-cube of $(\mathbf{n}-S)$-cubes in (9) is cartesian as a result.

The $S$-cube of $\mathbf{n}$-cubes obtained by letting $R^{\prime}$ in (9) vary over all subsets of $S$ is exactly (8). Since (9) is cartesian for each choice of $R^{\prime}$, Lemma 4.8 tells us that (8) is cartesian.

Finally, by assumption, we know that $\sqcup_{n} F(\widetilde{\mathbf{X}}(R,-))$ is a cartesian $n$-cube for each $R \neq S$. Hence, the fact that (8) is cartesian coupled with Lemma 4.8 again yields the fact that $\sqcup_{n} F(\widetilde{\mathbf{X}}(S,-))$ must be a cartesian $n$-cube as well.

With this, we prove Proposition 4.3 .

Proof. We begin by considering $\sqcup_{n} F(\widetilde{\mathbf{X}}(-,-))$ from the point of view of Remark 4.9 (2). Let $T \subset \mathbf{n}, T \neq \emptyset$, and $i \in T$. We can view $\sqcup_{n} F(\widetilde{\mathbf{X}}(-, T))$ as a map of two $(n-1)$-cubes

$$
\sqcup_{n} F(\widetilde{\mathbf{X}}(V, T)) \rightarrow \sqcup_{n} F(\widetilde{\mathbf{X}}(V \cup\{i\}, T)),
$$

where $V \subseteq S-\{i\}$. However, by definition, these two $(n-1)$-cubes are identical and the map between them is the identity. Hence, $\sqcup_{n} F(\widetilde{\mathbf{X}}(-, T))$ is cartesian for each $T \subseteq \mathbf{n}, T \neq \emptyset$.

Now assume that $F$ is degree $n-1$. By Remark 4.9)(2), it suffices to show that $\sqcup_{n} F(\widetilde{\mathbf{X}}(-, \emptyset))$ is cartesian to conclude that $F$ is $(n-1)$-excisive relative to $A$. 
Viewing the cube as in Remark $4.9(1)$, we see that for any $S \subseteq \mathbf{n}, \widetilde{\mathbf{X}}(S,-)$ is the $n$-cube $\left(\sqcup^{n}\right)_{B}^{\mathbf{Z}(S)}$ where $\mathbf{Z}(S)$ is the $n$-tuple whose $i$ th entry is

$$
\mathbf{Z}(S)_{i}= \begin{cases}X_{i} & \text { if } i \in S \\ A & \text { if } i \notin S\end{cases}
$$

Since $F$ is degree $n-1$, it follows that $\sqcup_{n} F(\widetilde{\mathbf{X}}(S,-))$ is cartesian. Then by Lemma 4.8. $\sqcup_{n} F(\widetilde{\mathbf{X}}(-,-))$ is cartesian. Since $\sqcup_{n} F(\widetilde{\mathbf{X}}(-, T))$ is cartesian for each $T \neq \emptyset$, Lemma 4.8 guarantees that $\sqcup_{n} F(\widetilde{\mathbf{X}}(-, \emptyset))$ must be as well.

Assuming that $F$ is $n$-excisive relative to $A$, by Remark 4.9(1), we need to show that $\sqcup_{n} F(\widetilde{\mathbf{X}}(\mathbf{n},-))$ is cartesian to conclude that $F$ is degree $n-1$. By Lemma 4.10, we know that $\sqcup_{n} F(\widetilde{\mathbf{X}}(S,-))$ is cartesian for each $S \subset \mathbf{n}, S \neq \mathbf{n}$. Hence by Lemma 4.8 it suffices to show that $\sqcup_{n} F(\widetilde{\mathbf{X}}(-,-))$ is cartesian. We do so by applying Lemma 4.8 after making sure that $\sqcup_{n} F(\widetilde{\mathbf{X}}(-, T))$ is cartesian for each $T \subseteq \mathbf{n}$. This has been done for $T \neq \emptyset$ above. When $T=\emptyset, \widetilde{\mathbf{X}}(-, T)$ is $\left(\sqcup^{n}\right)_{\mathbf{X}}^{A}$, and so $\sqcup_{n} F(\widetilde{\mathbf{X}}(-, T))$ is cartesian by assumption.

One can extend a functor $F: \mathcal{C}_{f} \rightarrow \mathcal{S}$ to the category of simplicial objects $s \mathcal{C}_{f}$ in two ways - by applying $F$ degreewise to a simplicial object $X$. to obtain a simplicial object $F(X$.) in $\mathcal{S}$ or by applying $F$ to the geometric realization $\mid X$. $\mid$ (recall our convention that all realizations are "fat"). When these two approaches agree, i.e., when the natural map $|F(X).| \rightarrow F(\mid X$. $\mid)$ is a weak equivalence for each simplicial object $X$., we say that $F$ commutes with realizations. In particular, by Remark 4.6, in $\mathcal{S}$, finite homotopy limits commute with realizations.

Proposition 4.11. If $F: \mathcal{C}_{f} \rightarrow \mathcal{S}$ commutes with realizations and preserves weak equivalences, then $F$ is degree $n$ if and only if $F$ is $n$-excisive.

Proof. By Proposition 4.3, we know that an $n$-excisive functor is always degree $n$, so we need only show that if $F$ is degree $n$, then $F$ is $n$-excisive. The strategy for the proof is to show that any strongly cocartesian $(n+1)$-cube $\mathcal{X}$ can be replaced by an equivalent $(n+1)$-cube $B(\mathcal{X})$ built using the generalized bar construction, as defined in Section 2.2. We are then able to show that $F(B(\mathcal{X}))$ is cartesian by applying Lemma 4.8 levelwise to a map of $(n+1)$-cubes $F\left(B^{-1}(\mathcal{X})\right) \rightarrow F(B(\mathcal{X}))$. We illustrate the case $n=1$ first, with the proof of $n>1$ to follow.

Let

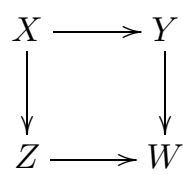

be a cocartesian square. Let $B_{\bullet}(X, Y)$ and $B_{\bullet}(X, Z)$ be the generalized bar constructions whose realizations yield $\operatorname{hocolim}(X \rightarrow Y)$ and $\operatorname{hocolim}(X \rightarrow Z)$, respectively. Since hocolim $\{X \rightarrow Y\} \simeq Y$, we know that $\left|B_{\bullet}(X, Y)\right| \simeq Y$. Similarly, $\left|B_{\bullet}(X, Z)\right| \simeq Z$, and $\left|B_{\bullet}(X)\right| \simeq X$, where $B_{\bullet}(X)$ is the generalized bar construction 
coming from the trivial diagram $X$. By the first two parts of Lemma 2.3.

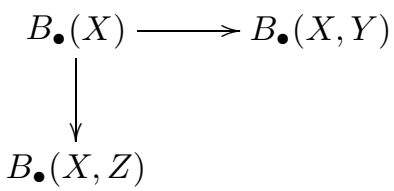

is a cofibrant replacement of the diagram

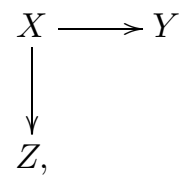

and hence we can take the strict pushout of this diagram in order to compute the homotopy colimit $W$. But by Lemma 2.3. (3), this strict pushout is precisely the bar construction whose realization yields $\operatorname{hocolim}(Y \leftarrow X \rightarrow Z)$. We use $B \bullet(Y, X, Z)$ to denote this generalized bar construction. Since $F$ preserves weak equivalences, we may replace our original diagram (12) with

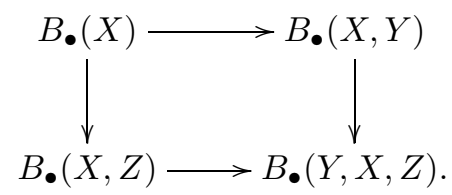

Looking levelwise we see that in degree $k$, the diagram is

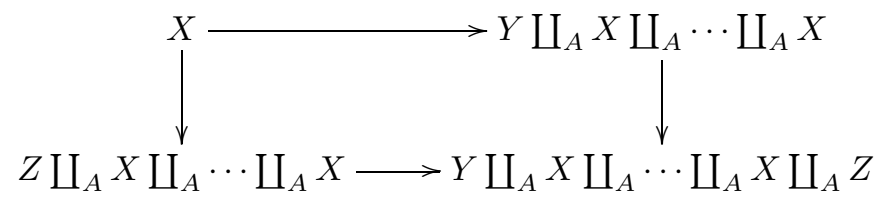

where the coproducts in the upper right and lower left corners contain $k+1$ copies of $X$ and in the bottom right corner has $2(k+1)-1$ copies of $X$.

After applying the degree 1 functor $F$ to (13), we would like to show that

$$
F\left(B_{\bullet}(X, Y, Z)\right)=B_{\bullet}(\tilde{F}(X), \tilde{F}(Y), \tilde{F}(Z))
$$

(where $\tilde{F}(-)$ represents the cofibrant replacement of $F(-)$ ). This is easily done in the degree 1 case because Proposition 4.3 guarantees that whenever $F$ is degree 1 , the diagram

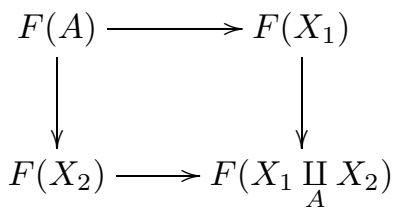

obtained by applying $F$ to a cocartesian square must be cartesian. Since $F$ takes values in $\mathcal{S}$, the square is also cocartesian, and so

$$
F\left(X_{1} \coprod_{A} X_{2}\right) \simeq F\left(X_{1}\right) \coprod_{F(A)} F\left(X_{2}\right) .
$$


However, this approach cannot be generalized to functors of degree $n>1$, so instead we will describe the proof for degree 1 with an eye towards the general case.

At each simplicial level, we want to expand (13) into the cube

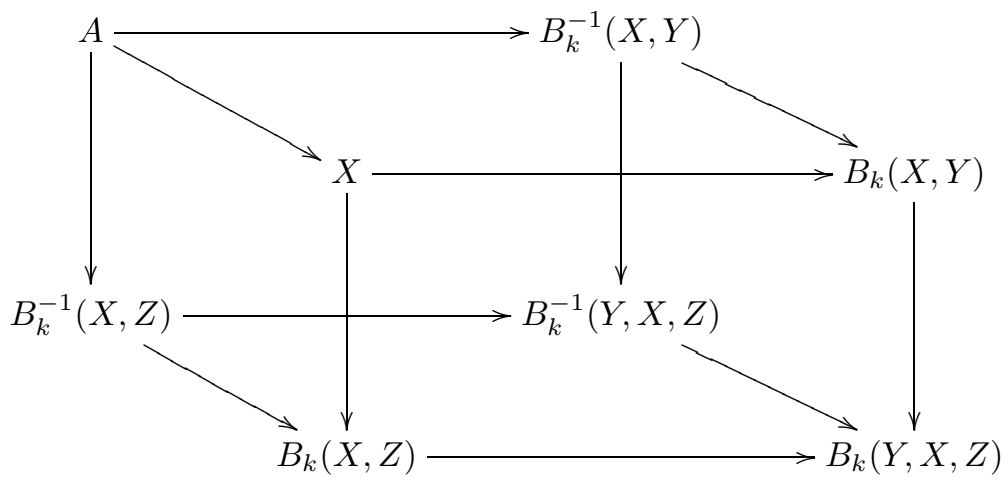

where $B_{k}(X, Y), B_{k}(X, Z)$ and $B_{k}(Y, X, Z)$ are the $k$ th levels of the simplicial sets described above, and $B_{k}^{-1}(X, Y)$ (respectively, $B_{k}^{-1}(X, Z)$ and $B_{k}^{-1}(Y, X, Z)$ ) is the object of $\mathcal{C}_{f}$ obtained from $B_{k}(X, Y)$ (respectively, $B_{k}(X, Z)$ and $B_{k}(Y, X, Z)$ ) by removing the copy of $X$ corresponding to the constant sequence $X=\cdots=X$. The map $B_{k}^{-1}(X, Y) \rightarrow B_{k}(X, Y)$ is then given by the natural inclusion (respectively, $B_{k}^{-1}(X, Z) \rightarrow B_{k}(X, Z)$ and $\left.B_{k}^{-1}(Y, X, Z) \rightarrow B_{k}(Y, X, Z)\right)$. We make no claim that $B_{\bullet}^{-1}(X, Y)$ is a simplicial object. Each square face of this cube is easily seen to be a strict pushout, so the cube is strongly cocartesian. As we determined in section 3.4, the fact that $F$ is degree 1 implies that it is also degree 2. Then by Proposition 4.3, we know that applying $F$ to (14) yields a cartesian diagram. The back face of (14),

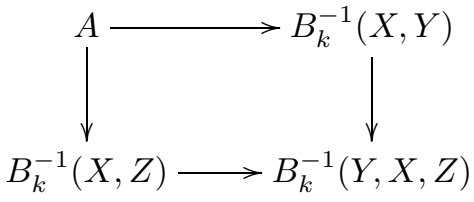

is a pushout diagram with initial vertex $A$, and since $F$ is degree 1 , Proposition 4.3 tells us that this square will be a cartesian square after $F$ is applied. Recognizing that $F$ takes values in $\mathcal{S}$, and applying Lemma 4.8, we can conclude that

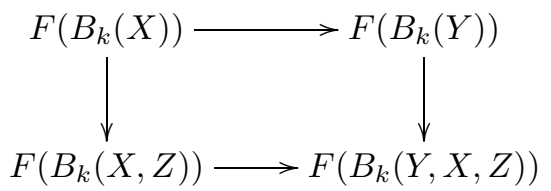

is also a pushout square.

We have now calculated that the diagram

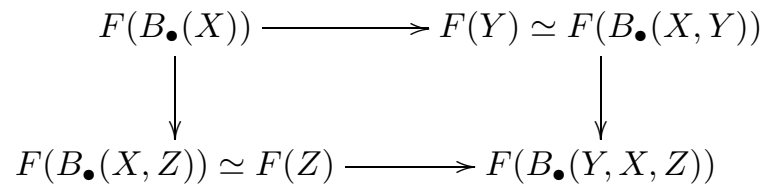


is a pushout diagram levelwise, and hence is a pushout diagram of simplicial sets. As a result,

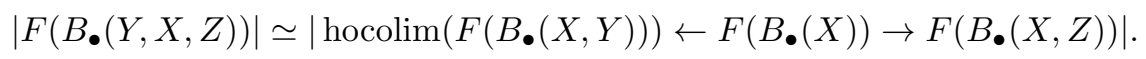

However, since $F$ commutes with realizations and hocolim commutes with realizations, the right hand side of this equivalence is equivalent to the homotopy pushout of $F(Y) \leftarrow F(X) \rightarrow F(Z)$.

To complete the proof, we note that by Remark 4.5 it suffices to show that applying $F$ to (12) yields a cocartesian square. Since $F$ commutes with realization we have

$$
\begin{gathered}
F(\operatorname{hocolim}\{Z \leftarrow X \rightarrow Y\}) \simeq F\left(\left|B_{\bullet}(Y, X, Z)\right|\right) \\
\simeq|F(B \bullet(Y, X, Z))| \simeq \operatorname{hocolim}\{F(Z) \leftarrow F(X) \rightarrow F(Y)\},
\end{gathered}
$$

which concludes the proof in the case $n=1$.

To prove the result for $n>1$, we let $\mathcal{X}: \mathcal{P}(\mathbf{n}) \rightarrow \mathcal{C}_{f}$ be a strongly cocartesian $n$-cube. For $S \in \mathcal{P}(\mathbf{n})$, let $\mathcal{D}_{S}$ be the restriction of $\mathcal{P}(\mathbf{n})$ to the collection of sets $\{\emptyset,\{s\} \mid s \in S\}$. Let $B_{\bullet}(\mathcal{X}, S)$ be the generalized bar construction computing $\operatorname{hocolim}_{\mathcal{D}_{S}}$. In simplicial level $k$,

$$
B_{k}(\mathcal{X}, S)=\coprod_{\alpha_{0} \rightarrow \cdots \rightarrow \alpha_{k} \in \mathcal{D}_{S}} \mathcal{X}\left(\alpha_{0}\right)
$$

where all coproducts are taken over $\mathcal{X}(\emptyset)$. We claim that the $n$-cube $\mathcal{X}$ is weakly equivalent to the $n$-cube $B_{\bullet}(\mathcal{X},-)$. One can verify this by using the fact that $\mathcal{X}$ is strongly cocartesian and by applying Lemma 2.3(3). In particular, if $S, S^{\prime}$ are subsets of $\mathbf{n}$, then

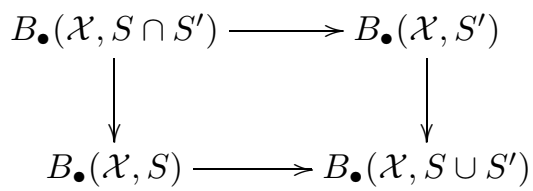

is a pushout diagram.

Now, for each $k$ we consider the $(n+1)$-cube $B_{k}^{-1}(\mathcal{X},-) \rightarrow B_{k}(\mathcal{X},-)$, where $B_{k}^{-1}(\mathcal{X}, S)$ is obtained from $B_{k}(\mathcal{X}, S)$ by removing the copy of $\mathcal{X}(\emptyset)$ corresponding to the constant sequence $\emptyset \rightarrow \cdots \rightarrow \emptyset$ for each $\emptyset \neq S \subset \mathbf{n}$, and $B_{k}^{-1}(\mathcal{X}, \emptyset)=A$. There are natural inclusion maps $B_{k}^{-1}(\mathcal{X}, S) \rightarrow B_{k}(\mathcal{X}, S)$ given by the inclusion $A \rightarrow X$ indexed by the sequence $\emptyset \rightarrow \cdots \rightarrow \emptyset$. The $n$-cube $B_{k}^{-1}(\mathcal{X}, S)$ is again strongly cocartesian. For any $S \rightarrow S \cup\{i\}$ in $\mathcal{P}(\mathbf{n})$, the diagram

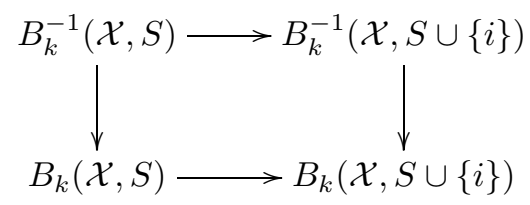

is easily seen to be a pushout diagram, since it can be rewritten as

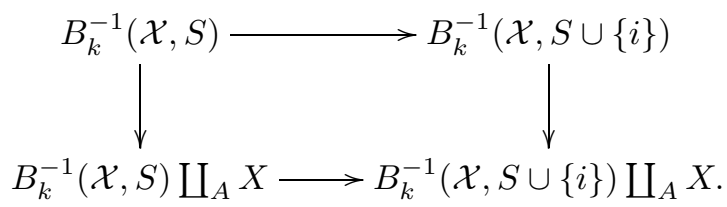


Thus the $(n+1)$-cube $B_{k}^{-1}(\mathcal{X}, S) \rightarrow B_{k}(\mathcal{X}, S)$ and the $n$-cube $B_{k}^{-1}(\mathcal{X}, S)$ are strongly cocartesian cubes with initial vertex $A$, and any degree $n-1$ functor $F$ will take these to cartesian cubes by Proposition 4.3. The remainder of the proof now proceeds exactly as in the case $n=1$.

\section{A TAYLOR TOWER FROM COTRIPLES}

Having established that $\left(\perp_{n}, \delta, \epsilon\right)$ is a cotriple for $n \geq 1$, we show in this section how to define the cotriple Taylor tower for a functor from $\mathcal{C}_{f}$ to $\mathcal{S}$ where $\mathcal{C}_{f}$ and $\mathcal{S}$ are as described in the beginning of Sections 3 and 4 . The results in this section are generalizations to $\mathcal{C}_{f}$ and $\mathcal{S}$ of results in Section 2 of [13. In the next section of this paper, we compare this cotriple Taylor tower to Goodwillie's Taylor tower of $n$-excisive approximations.

To define the terms in our Taylor tower, we use the augmented simplicial objects associated to the cotriples $\left(\perp_{n}, \delta, \epsilon\right)$.

Definition 5.1. Let $F$ be a functor from $\mathcal{C}_{f}$ to $\mathcal{S}$ and $n \geq 1$. We use $\perp_{n}^{*+1} F$ to denote the simplicial object constructed from $F$ using the cotriple $\left(\perp_{n}, \delta, \epsilon\right)$. More specifically, $\perp_{n}^{*+1} F$ is the simplicial object that in simplicial degree $k$ is $\perp_{n}^{k+1} F$ with face and degeneracy maps defined by

$$
\begin{gathered}
s_{i}=\perp^{i} \delta \perp^{k-i}: \perp_{n}^{k} F \rightarrow \perp_{n}^{k+1} F, \\
d_{i}=\perp^{i} \epsilon \perp^{k-i}: \perp_{n}^{k} F \rightarrow \perp_{n}^{k-1} .
\end{gathered}
$$

Remark 5.2. We note that $\perp_{n}^{*+1}$ is augmented over $\operatorname{id}_{\operatorname{Fun}\left(\mathcal{C}_{f}, \mathcal{S}\right)}$ by $\epsilon$. Hence, $\epsilon$ yields a natural simplicial map from $\perp_{n}^{*+1}$ to the simplicial object $\mathrm{id}^{*+1}$ associated to the identity cotriple $\left(\operatorname{id}_{\operatorname{Fun}\left(\mathcal{C}_{f}, \mathcal{S}\right)}\right.$, id, id) built out of the identity functor.

Definition 5.3. Let $F$ be a functor from $\mathcal{C}_{f}$ to $\mathcal{S}$ and $n \geq 0$. The $n$th term in the cotriple Taylor tower of $F$ is the functor

$$
\Gamma_{n} F:=\operatorname{hocofiber}\left(\left|\perp_{n+1}^{*+1} F\right| \stackrel{\widehat{\epsilon}}{\longrightarrow} F\right)
$$

(recall our convention that realizations are "fat"), where the map

$$
\left|\perp_{n+1}^{*+1} F\right| \stackrel{\widehat{\epsilon}}{\longrightarrow} F
$$

is the composition of the map induced by $\epsilon$ with the weak equivalence

$$
\left|\operatorname{id}^{*+1} F\right| \stackrel{\simeq}{\longrightarrow} F
$$

and hocofiber denotes the homotopy cofiber given by

$$
\operatorname{hocofiber}(A \rightarrow B):=\operatorname{hocolim}(* \longleftarrow A \longrightarrow B) \text {. }
$$

We use $\gamma_{n} F$ to denote the natural transformation $F \rightarrow \Gamma_{n} F$ in the resulting cofibration sequence $\left|\perp_{n+1}^{*+1} F\right| \rightarrow F \rightarrow \Gamma_{n} F$.

Convention. Even when $F$ takes fibrant values, $\Gamma_{n} F$ may not. Since $\mathcal{S}$ has functorial fibrant replacements, we can replace $\Gamma_{n} F$ by a fibrant-valued, weakly equivalent functor $\hat{\Gamma}_{n} F$, in which case we actually obtain a natural diagram

$$
F \longrightarrow \Gamma_{n} F \ll \hat{\Gamma}_{n} F .
$$

We will abuse notation and continue to write this as $F \rightarrow \Gamma_{n} F$ and assume without loss of generality that our $\Gamma_{n} F$ takes fibrant values. 
Using properties of adjoint pairs, one can show that $\Gamma_{n} F$ is a degree $n$ approximation to $F$.

Proposition 5.4. For a functor $F: \mathcal{C}_{f} \rightarrow \mathcal{S}$, the functor $\Gamma_{n} F$ is degree $n$.

The analogous statement for functors to abelian categories, in 13 (Lemma 2.11), was proved by establishing that $\perp_{n+1} \perp_{n+1}^{*+1} F \rightarrow \perp_{n+1} F$ has a simplicial homotopy inverse, and hence, $\perp_{n+1} \Gamma_{n} F$ is contractible. In the abelian setting, this was enough to conclude that for any collection of objects, $X_{1}, \ldots, X_{n+1}$, in the domain category, $c r_{n+1} \Gamma_{n} F\left(X_{1}, \ldots, X_{n+1}\right)$ is contractible, since it is a direct summand of $\perp_{n+1} \Gamma_{n} F\left(\coprod X_{i}, \ldots, \coprod X_{i}\right)$. In the present paper, this last step is not an option. However, one can modify the homotopies used in the abelian case to prove directly that

$$
c r_{n+1} \perp_{n+1}^{*+1} F \rightarrow c r_{n+1} F
$$

is a homotopy equivalence. We adapt this approach for use in this paper.

The proof makes use of the following general facts about adjoint pairs of functors.

Lemma 5.5. Let $(L, R): \mathcal{A} \rightarrow \mathcal{B}$ be a pair of adjoint functors where $L: \mathcal{A} \rightarrow \mathcal{B}$ is the left adjoint. Let $\perp=L R: \mathcal{B} \rightarrow \mathcal{B}$ be the associated cotriple and $B$ be an object in $\mathcal{B}$. Then there are natural simplicial maps $f: R\left(\mathrm{id}^{*+1} B\right) \rightarrow R\left(\perp^{*+1} B\right)$ and $v: R\left(\perp^{*+1} B\right) \rightarrow R\left(\mathrm{id}^{*+1} B\right)$ such that $f \circ v$ is naturally homotopic to the identity on $R\left(\perp^{*+1} B\right)$ and $v \circ f$ is naturally homotopic to the identity on $R\left(\mathrm{id}^{*+1} B\right)$.

Proof. Let $\eta: \operatorname{id}_{\mathcal{A}} \rightarrow R L$ be the unit of the adjunction and $\epsilon: L R \rightarrow \mathrm{id}_{\mathcal{B}}$ be the counit of the adjunction. One can use $\epsilon$ and $\eta$ to construct the simplicial maps $f: R\left(\mathrm{id}^{*+1} B\right) \rightarrow R\left(\perp^{*+1} B\right)$ and $v: R\left(\perp^{*+1} B\right) \rightarrow R\left(\mathrm{id}^{*+1} B\right)$, and simplicial homotopies between $f \circ v$ and $v \circ f$ and the appropriate identity maps. In particular, in degree $k, v_{k}=R\left(\epsilon \circ \epsilon_{\perp} \circ \cdots \circ \epsilon_{\perp^{k}}\right)$ and $f_{k}=\eta_{R(L R)^{k}} \circ f_{k-1}$ with $f_{0}=\eta_{R}$. One can construct the homotopies in a similar fashion to that of Exercise 8.3.7 of [22], using $\eta_{R}$ in place of the extra degeneracy $\sigma_{0}$.

With this, we prove Proposition 5.4

Proof. Applying the above to the adjoint pair $\left(\Delta^{*} \circ U^{+}, t^{+} \circ \sqcup_{n+1}\right)$ of the previous section, we see that $\left(t^{+} \circ \sqcup_{n+1}\right) \perp_{n+1}^{*+1} F$ is weakly equivalent to $t^{+} \circ \sqcup_{n+1} F$ via the augmentation $t^{+} \circ \sqcup_{n+1}(\epsilon)$. Setting $c r_{n+1}^{+} F=t^{+} \circ \sqcup_{n+1} F$, this becomes

$$
c r_{n+1}^{+}\left(\perp_{n+1}^{*+1} F\right) \simeq c r_{n+1}^{+}\left(\mathrm{id}^{*+1} F\right) .
$$

Applying the forgetful functor from $\operatorname{Fun}\left(\mathcal{C}_{f}^{\times n+1}, \mathcal{S}\right)_{t}$ to $\operatorname{Fun}\left(\mathcal{C}_{f}^{\times n+1}, \mathcal{S}\right)$ (this functor "forgets" the coalgebra structure) gives us an equivalence between $c r_{n+1} F$ and $c r_{n+1} \perp_{n+1} F$. The fact that $c r_{n+1}$, as a finite homotopy limit, commutes with finite and filtered homotopy colimits implies that

$$
\begin{aligned}
c r_{n+1} \Gamma_{n} F & =c r_{n+1}\left(\text { hocofiber }\left(\left|\perp_{n+1}^{*+1} F\right| \rightarrow\left|\mathrm{id}^{*+1} F\right|\right)\right) \\
& \simeq \operatorname{hocofiber}\left(\left|c r_{n+1}\left(\perp_{n+1}^{*+1} F\right)\right| \rightarrow\left|c r_{n+1}\left(\mathrm{id}^{*+1} F\right)\right|\right) \\
& \simeq \star
\end{aligned}
$$

Hence, $\Gamma_{n} F$ is degree $n$.

We consider the functor $\Gamma_{n} F$ to be an "approximation" to $F$ in the following sense. 
Proposition 5.6. (1) If $F$ is degree $n$, then the natural transformation $\gamma_{n} F$ : $F \rightarrow \Gamma_{n} F$ is a weak equivalence.

(2) The pair $\left(\Gamma_{n} F, \gamma_{n} F\right)$ is universal, up to weak equivalence, among degree $n$ functors with natural transformations from $F$.

The proofs of these results are similar to those of Proposition 1.18 in [10] and Lemma 2.11 in 13 and are omitted.

We end this section by defining natural transformations $\Gamma_{n} F \rightarrow \Gamma_{n-1} F$ that allow us to assemble the $\Gamma_{n} F$ s into a Taylor tower for $F$.

Definition 5.7. Let $X$ be an object in $\mathcal{C}_{f}$. We define the $(n+1)$-cube of $n$-tuples, $\mathbf{X}^{\prime}: \mathcal{P}(\mathbf{n}) \rightarrow \mathcal{C}_{f}^{\times n}$, as follows. For $i \neq 1$ and $S \subseteq \mathbf{n}+\mathbf{1}$, the $i$ th entry in $\mathbf{X}^{\prime}(S)$ is

$$
\mathbf{X}^{\prime}(S)_{i}= \begin{cases}X & \text { if }\{2, i+1\} \cap S=\emptyset \\ B & \text { otherwise }\end{cases}
$$

and, for $i=1$, we have

$$
\mathbf{X}^{\prime}(S)_{1}= \begin{cases}X \coprod_{A} X & \text { if }\{1,2\} \cap S=\emptyset, \\ B \coprod_{A} B & \text { otherwise. }\end{cases}
$$

The morphisms are all induced by the morphism $X \rightarrow B$.

Recalling the definition of $\left(\sqcup^{n+1}\right)_{B}^{(X, X, \ldots, X)}$ from Example 2.8 it is easy to verify that there is a natural map of $(n+1)$-cubes $\tau_{n}:\left(\sqcup^{n+1}\right)_{B}^{(X, X, \ldots, X)} \rightarrow \sqcup^{n} \mathbf{X}^{\prime}$. Applying a functor $F$ and taking the total fiber of the resulting $(n+1)$-cubes, yields a natural map

$$
\rho_{n}: \perp_{n+1} F(X) \rightarrow \text { tfiber } F\left(\sqcup^{n} \mathbf{X}^{\prime}\right) .
$$

But, by construction, letting $\mathbf{X}^{\prime \prime}$ denote the restriction of $\mathbf{X}^{\prime}$ to $S \subseteq \mathbf{n}+\mathbf{1}$ with $2 \notin S$, we see that

$$
\text { tfiber } \begin{aligned}
F\left(\sqcup^{n} \mathbf{X}^{\prime}\right) & \simeq \text { hofiber }\left(\operatorname{tfiber} F\left(\sqcup^{n} \mathbf{X}^{\prime \prime}(-)\right) \rightarrow \operatorname{tfiber} F\left(\sqcup^{n} \mathbf{X}^{\prime \prime}(-\cup\{2\})\right)\right) \\
& \simeq \operatorname{hofiber}\left(\operatorname{tfiber} F\left(\sqcup^{n} \mathbf{X}^{\prime \prime}(-)\right) \rightarrow \star\right) \\
& \simeq \operatorname{tfiber} F\left(\sqcup^{n} \mathbf{X}^{\prime \prime}(-)\right),
\end{aligned}
$$

and we have a natural map

$$
\iota_{n}: \text { tfiber } F\left(\sqcup^{n} \mathbf{X}^{\prime}\right) \stackrel{\simeq}{\longrightarrow} \text { tfiber } F\left(\sqcup^{n} \mathbf{X}^{\prime \prime}\right) .
$$

Applying the fold map $+: Y \coprod_{A} Y \rightarrow Y$ (with $Y$ equal to $X$ or $B$ ) to the first pair of terms in the coproducts produces a natural map of $n$-cubes that yields

$$
\sigma_{n}: \text { tfiber } F\left(\sqcup^{n} \mathbf{X}^{\prime \prime}\right) \rightarrow \perp_{n} F(X) .
$$

We define $\nu_{n}: \perp_{n+1} F(X) \rightarrow \perp_{n} F(X)$ to be the natural composition

$$
\nu_{n}=\sigma_{n} \circ \iota_{n} \circ \rho_{n} .
$$

This gives us a map of simplicial objects $\perp_{n+1}^{*} F \rightarrow \perp_{n}^{*} F$ that can be used to construct the natural transformation $q_{n} F: \Gamma_{n} F \rightarrow \Gamma_{n-1} F$. With this map we obtain the desired Taylor tower. 
Theorem 5.8. There is a natural tower of functors:

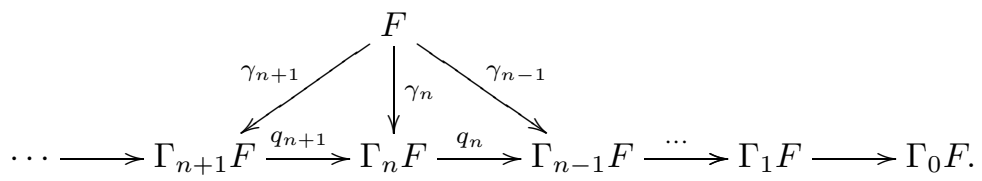

\section{A COMPARISON to GoOdWILliE's n-EXCISIVE APPROXIMATION}

We use this section to compare the degree $n$ approximation, $\Gamma_{n} F$, of a functor $F: \mathcal{C}_{f} \rightarrow \mathcal{S}$ to Goodwillie's $n$-excisive approximation, $P_{n} F$. After reviewing the definition of $P_{n} F$, we first show that when $F$ commutes with realizations, $P_{n} F$ and $\Gamma_{n} F$ are weakly equivalent as functors from $\mathcal{C}_{f}$ to $\mathcal{S}$. When $F$ does not commute with realizations, we also obtain an agreement of the functors $\Gamma_{n} F$ and $P_{n} F$, but only when evaluated at the initial object of $\mathcal{C}_{f}$. We conclude the section by using this fact to show that for an object $X$, the degree $n$ and $n$-excisive approximations to $F$ at $X$ agree, but only after restricting the constructions (in a sense to be made clear later) to $\mathcal{C}_{\beta: X \rightarrow B}$. Throughout this section $\mathcal{C}$ and $\mathcal{D}$ are used to denote simplicial model categories, and $\mathcal{S}$ is a suitable model of spectra as in section 4 . As in previous sections, for a morphism $f: A \rightarrow B$ in $\mathcal{C}$, we use $\mathcal{C}_{f}$ to denote the category of objects of $\mathcal{C}$ that factor $f$, and we use $\star$ to denote the initial/final object in $\mathcal{S}$. While the proofs of the two main theorems of the section require that our functors take values in $\mathcal{S}$, some of the lemmas used to prove them are stated and proved more generally for functors from $\mathcal{C}$ to $\mathcal{D}$.

6.1. Goodwillie's $n$-excisive approximations. To define the $n$-excisive approximation to a functor $F$, we make use of the following construction for objects $X$ in $\mathcal{C}_{f}$.

Definition 6.1. For a finite set $U$ of cardinality $u$, let $\mathcal{D}_{U}$ be the poset obtained from $\mathcal{P}(U)$ by restricting to the empty set and one element subset of $U$. That is, the set of objects of $\mathcal{D}_{U}$ is $\{\emptyset,\{t\} \mid t \in U\}$. For an object $X$ in $\mathcal{C}_{f}$ with $\beta: X \rightarrow B$, we define a functor $X_{U}: \mathcal{D}_{U} \rightarrow \mathcal{C}_{f}$ by

$$
X_{U}(S)= \begin{cases}X & \text { if } S=\emptyset, \\ B & \text { if } S \neq \emptyset,\end{cases}
$$

and $X_{U}(\emptyset \rightarrow\{t\})=\beta$. We define $B \otimes_{X}-$ to be the functor from finite sets to $\mathcal{C}_{f}$ that for the finite set $U$ is given by

$$
B \otimes_{X} U:=\underset{S \in \mathcal{D}_{U}}{\operatorname{hocolim}} X_{U}(S) .
$$

Remark 6.2. Suppose that $U$ has $u$ elements.

(1) If we assume that $\beta: X \rightarrow B$ is a cofibration, then $B \otimes_{X} U$ is the coproduct over $X$ of $u$ copies of $B$. In particular, if $U$ is the empty set, then $B \otimes_{X} U \simeq$ $X$, and if $U$ has a single element, then $B \otimes_{X} U \simeq B$.

(2) Let $\mathcal{U}_{Y}$ be the category of unbased spaces over a fixed space $Y$ used by Goodwillie. The initial and final objects are $\emptyset$ and $Y$, respectively. When $X$ is an object in $\mathcal{U}_{Y}$, the space $Y \otimes_{X} U$ is equivalent to the fibrewise join of $X$ with $U$ over $Y$, denoted $X *_{Y} U$, that Goodwillie uses to define $P_{n} F$. Recall that $X *_{Y} U$ is defined as

$$
\operatorname{hocolim}(X \leftarrow X \times U \rightarrow Y \times U) \text {. }
$$


One can see that $Y \otimes_{X} U$ and $X *_{Y} U$ are equivalent either by directly comparing the homotopy colimits used to define them or by noting that both constructions yield $u$ copies of the mapping cone of $X \rightarrow Y$ identified together along a single copy of $X$.

To define $P_{n} F(X)$ for a functor of $\mathcal{U}_{Y}$ and object $X$ in $\mathcal{U}_{Y}$, Goodwillie defines an intermediate functor $T_{n} F$, by

$$
T_{n} F(X):=\operatorname{holim}_{U \in \mathcal{P}_{0}(\mathbf{n}+\mathbf{1})} F\left(X *_{Y} U\right) .
$$

There is a natural map $F(X) \simeq F\left(X *_{Y} \emptyset\right) \rightarrow T_{n} F(X)$, and iterating yields a sequence

$$
F(X) \rightarrow T_{n} F(X) \rightarrow T_{n}^{2} F(X) \rightarrow T_{n}^{3} F(X) \rightarrow \ldots
$$

The functor $P_{n} F$ is defined as the homotopy colimit of this sequence:

$$
P_{n} F(X):=\operatorname{hocolim}_{k} T_{n}^{k} F(X) .
$$

See section 1 of [10] for more details.

In light of Remark 6.2(2), we extend Goodwillie's definition of $P_{n} F$ to functors from $\mathcal{C}_{f}$ to $\mathcal{D}$ as follows.

Definition 6.3. Let $F: \mathcal{C}_{f} \rightarrow \mathcal{D}$, and let $X$ be an object in $\mathcal{C}_{f}$ (with canonical map $\beta: X \rightarrow B)$. Let $T_{n} F(X)$ be defined by

$$
T_{n} F(X):=\operatorname{holim}_{U \in \mathcal{P}_{0}(\mathbf{n}+\mathbf{1})} F\left(B \otimes_{X} U\right) .
$$

Then $P_{n} F(X)$ is given by

$$
P_{n} F(X):=\operatorname{hocolim}_{k}\left(T_{n}^{k} F(X)\right) .
$$

N. Kuhn has also defined $P_{n} F$ for functors of pointed simplicial or topological model categories ([14). As an immediate consequence of Definition 6.3, we have the next lemma.

Lemma 6.4. For $F$ a functor from $\mathcal{C}_{f}$ to $\mathcal{S}$, the construction $P_{n}$ satisfies the following properties:

(1) Let $\left\{F_{i}\right\}, i \in I$, be a finite diagram of functors from $\mathcal{C}_{f}$ to $\mathcal{S}$. Then

$$
\underset{i \in I}{\operatorname{holim}} P_{n} F_{i} \simeq P_{n}\left(\operatorname{holim}_{i \in I} F_{i}\right)
$$

as functors from $\mathcal{C}_{f}$ to $\mathcal{S}$.

(2) Let $G$. be a simplicial object in the category of functors from $\mathcal{C}_{f}$ to $\mathcal{S}$. Then

$$
P_{n}|G .| \simeq\left|P_{n} G .\right|,
$$

where $P_{n}$ is applied levelwise to $G$.

(3) Cofibration sequences of functors from $\mathcal{C}_{f}$ to $\mathcal{S}$ are preserved by $P_{n}$.

(4) For $F: \mathcal{C}_{f} \rightarrow \mathcal{D}$, there is natural transformation $F \rightarrow P_{n} F$. When $F$ is $n$-excisive, this is a weak equivalence.

Proof. The first part of the lemma follows from the definition of $P_{n}$ and the fact that homotopy limits commute and filtered countable homotopy colimits commute with finite homotopy limits in $\mathcal{S}$. To see that the second part is true, note that the fact that homotopy colimits commute tells us that for an object $X$ in $\mathcal{C}_{f}$,

$$
\left|P_{n} G .(X)\right|:=\left|\operatorname{hocolim}_{k} T_{n}^{k} G .(X)\right| \simeq \operatorname{hocolim}_{k}\left|T_{n}^{k} G .(X)\right| \text {. }
$$


So, it remains to show that

$$
\left|T_{n}^{k} G \cdot(X)\right| \simeq T_{n}^{k}|G \cdot|(X),
$$

where, by definition, $T_{n}^{k}|G|.(X)$ is the finite homotopy limit of a diagram of spectra. In general, homotopy limits do not commute with (fat) realizations, but in this case we have a finite homotopy limit in $\mathcal{S}$. The third part is proved using similar arguments.

The natural transformation of the fourth part is the transformation from $F$, the initial object of the sequence defining $P_{n} F$, into the homotopy colimit of that sequence. To understand what happens when $F$ is $n$-excisive, note that for an object $X$, the $(n+1)$-cube

$$
U \in \mathcal{P}(\mathbf{n}+\mathbf{1}) \mapsto B \otimes_{X} U
$$

is a strongly cocartesian diagram. Applying $F$ yields a cartesian diagram, and so we have an equivalence

$$
F\left(B \otimes_{X} \emptyset\right) \stackrel{\simeq}{\longrightarrow} \operatorname{holim}_{U \in \mathcal{P}_{0}(\mathbf{n}+\mathbf{1})} F\left(B \otimes_{X} U\right),
$$

but this is simply $F(X) \stackrel{\simeq}{\longrightarrow} T_{n} F(X)$. The result follows.

6.2. Functors that commute with realizations. Given a functor $F: \mathcal{C}_{f} \rightarrow \mathcal{S}$ we seek to show that $P_{n} F$ and $\Gamma_{n} F$ agree as functors of $\mathcal{C}_{f}$ when $F$ commutes with realizations. This is achieved via the next theorem.

Theorem 6.5. Let $F: \mathcal{C}_{f} \rightarrow \mathcal{S}$ be a functor that commutes with realizations. Then there is a (co)fibration sequence of functors

$$
\left|\perp_{n+1}^{*+1} F\right| \rightarrow F \rightarrow P_{n} F .
$$

Proof. Consider the cofibration sequence used to define $\Gamma_{n} F$ :

$$
\left|\perp_{n+1}^{*+1} F\right| \rightarrow F \rightarrow \Gamma_{n} F .
$$

Applying $P_{n}$ to the cofibration sequence and using the natural transformation of Lemma 6.4(4) gives us the commutative diagram below:

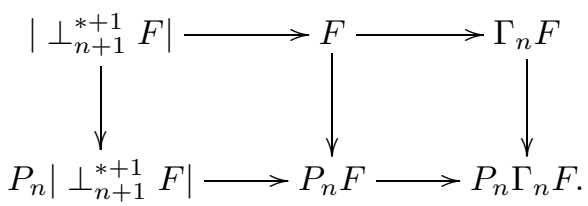

Both rows of this diagram are cofibration sequences, the first by definition, and the second because $P_{n}$ preserves cofibrations. Consider the top row of the diagram. By definition, $\perp_{n+1} F(X)=\operatorname{tfiber}\left(F\left(\sqcup^{n}\right)_{B}^{(X, \ldots, X)}\right)$. Since $\perp_{n+1} F$ is formed by a finite homotopy inverse limit, and since $F$ commutes with realizations and finite homotopy inverse limits in $\mathcal{S}$ commute with realizations, $\perp_{n+1} F$ commutes with realizations as well. As a result, we see that each functor in the top row commutes with realizations. In particular, $\Gamma_{n} F$ commutes with realizations, and so, by Propositions 4.11 and 5.4. $\Gamma_{n} F$ is $n$-excisive. By Lemma 6.4(4), the rightmost map in 
(15) is an equivalence. This tells us that the square

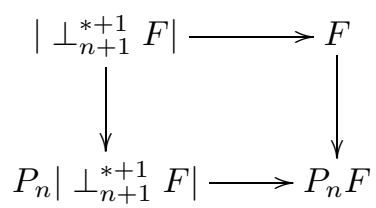

is cocartesian. To finish the proof, we show $P_{n}\left|\perp_{n+1}^{*+1} F\right| \simeq \star$, the initial/final object in $\mathcal{S}$. To do so, note that Lemma 6.4 (2) gives us

$$
P_{n}\left|\perp_{n+1}^{*+1} F\right| \simeq\left|P_{n} \perp_{n+1}^{*+1} F\right|,
$$

and so it is enough to show that $P_{n} \perp_{n+1}^{*+1} F \simeq \star$ by proving that $P_{n} \perp_{n+1} F \simeq \star$. We do so in Corollary 6.7 below.

To prove Corollary 6.7 we use the following lemma, which is Lemma 3.1 of [10]. In stating the lemma we use the notion of weakly $n$-reduced functors and the functor $\Delta^{*}$ from Definitions 3.18 and 3.15 .

Lemma 6.6. Let $G: \mathcal{C}_{f}^{\times n} \rightarrow \mathcal{D}$ be a weakly $n$-reduced functor. Then $P_{n-1} \Delta^{*} G \simeq \star$.

Proof. We prove this by first showing that the natural transformation from $\Delta^{*} G$ to $T_{n-1} \Delta^{*} G$ factors through $\star$. To do so, for an object $X \in \mathcal{C}_{f}$, we consider the $n$-cube $\widetilde{G}(X)$ :

$$
U \in \mathcal{P}(\mathbf{n}) \mapsto \widetilde{G}(X)(U)=G\left(X_{1}(U), X_{2}(U), \ldots, X_{n}(U)\right),
$$

where

$$
X_{i}(U)= \begin{cases}B \otimes_{X} \emptyset \simeq X & \text { if } i \notin U, \\ B \otimes_{X}\{i\} \simeq B & \text { if } i \in U .\end{cases}
$$

We also consider the $n$-cube $\widetilde{T} G(X)$ used to define $T_{n-1} \Delta^{*} G(X)$ :

$$
U \in \mathcal{P}(\mathbf{n}) \mapsto \widetilde{T} G(X)(U)=\Delta^{*} G\left(B \otimes_{X} U\right) .
$$

The inclusions $\{i\} \rightarrow U$ and $\emptyset \rightarrow U$ induce a map of $n$-cubes $\widetilde{G}(X) \rightarrow \widetilde{T} G(X)$. Moreover, the map $\Delta^{*} G(X) \rightarrow T_{n-1} \Delta^{*} G(X)$ factors as

$$
\begin{aligned}
\Delta^{*} G(X) \simeq \widetilde{G}(X)(\emptyset) & \rightarrow \operatorname{holim}_{U \in \mathcal{P}_{0}(\mathbf{n})} \widetilde{G}(X)(U) \\
& \rightarrow \operatorname{holim}_{U \in \mathcal{P}_{0}(\mathbf{n})} \widetilde{T} G(X)(U) \simeq T_{n-1} \Delta^{*} G(X) .
\end{aligned}
$$

Since $G$ is weakly $n$-reduced, $\widetilde{G}(X)(U) \simeq \star$ for $U \neq \emptyset$, and so we see that $\Delta^{*} G(X) \rightarrow T_{n-1} \Delta^{*} G(X)$ factors through $\star$. In a similar fashion, we can show that $T_{n-1}^{k} \Delta^{G}(X) \rightarrow T_{n-1}^{k+1} \Delta^{G}(X)$ factors through $\star$ for all $k$ and obtain the result.

Noting that $\perp_{n+1} F=\Delta^{*} c r_{n+1} F$, where $c r_{n+1} F$ is a weakly $(n+1)$-reduced functor (by Corollary 3.20), we obtain the desired corollary.

Corollary 6.7. For a functor $F: \mathcal{C}_{f} \rightarrow \mathcal{D}, P_{n} \perp_{n+1} F \simeq \star$.

The following corollary is an immediate consequence of Theorem 6.5.

Corollary 6.8. Let $F: \mathcal{C}_{f} \rightarrow \mathcal{S}$ be a functor that commutes with realizations. Then $P_{n} F$ and $\Gamma_{n} F$ are weakly equivalent as functors from $\mathcal{C}_{f}$ to $\mathcal{S}$. 
6.3. Functors that do not commute with realizations. If $F$ does not commute with realizations, the functors $P_{n} F$ and $\Gamma_{n} F$ no longer agree on all objects in $\mathcal{C}_{f}$, but they still agree at the initial object $A$. We establish this fact in the next theorem.

Theorem 6.9. Let $F: \mathcal{C}_{f} \rightarrow \mathcal{S}$, where $\mathcal{C}_{f}$ is the category of objects factoring the morphism $f: A \rightarrow B$. Then $\Gamma_{n} F(A) \simeq P_{n} F(A)$.

We prove this theorem in a manner similar to that of Theorem 6.5 and its corollary. In particular, we use the commutative diagram (15) evaluated at $A$ :

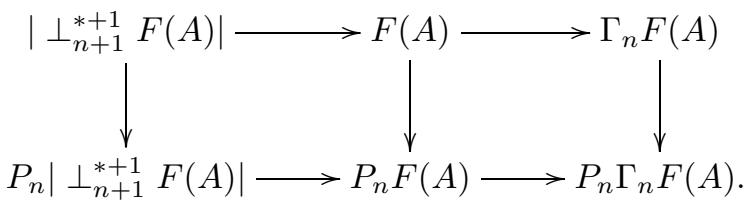

In this case, we will use the following lemmas to prove that the right vertical map is an equivalence and that $P_{n}\left|\perp_{n+1}^{*+1} F(A)\right| \simeq \star$. The second lemma is a consequence of the key observation in the first lemma.

Lemma 6.10. Let $F: \mathcal{C}_{f} \rightarrow \mathcal{S}$. Then

$$
\perp_{n+1} F(A) \rightarrow F(A) \rightarrow T_{n} F(A)
$$

is a fibration sequence in $\mathcal{S}$. B.

A generalization of this result for $T_{n}^{k} F(A), k \geq 1$, is the main result of Appendix

Proof. Recall our assumption that $A \stackrel{f}{\rightarrow} B$ is a cofibration. Because we are evaluating at the initial object in $\mathcal{C}_{f}$, the coproducts used to define $T_{n} F(A)$ are taken over $A$, as are the coproducts used in the $(n+1)$-cubical diagram whose total fiber defines $\perp_{n+1} F$. In particular, $\perp_{n+1} F(A)$ is the total fiber of the $(n+1)$-cubical diagram that assigns the object $F\left(A_{1}(S) \amalg \cdots \coprod A_{n+1}(S)\right)$ to the set $S$ where

$$
A_{i}(S)= \begin{cases}A & \text { if } i \notin S, \\ B & \text { if } i \in S .\end{cases}
$$

Since the coproducts are taken over $A$, letting $s$ denote the cardinality of $S$, we have

$$
A_{1}(S) \coprod \cdots \coprod A_{n}(S) \simeq \overbrace{B \coprod_{A} \cdots \coprod_{A} B}^{s \text { times }} \simeq B \otimes_{A} S .
$$

Then, by Remark 4.7 .

$$
\begin{aligned}
\perp_{n+1} F(A) & \simeq \operatorname{tfiber}\left(S \in \mathcal{P}(\mathbf{n}+\mathbf{1}) \mapsto F\left(A_{1}(S) \amalg \cdots \amalg A_{n+1}(S)\right)\right) \\
& \simeq \operatorname{tfiber}\left(S \in \mathcal{P}(\mathbf{n}+\mathbf{1}) \mapsto F\left(B \otimes_{A} S\right)\right) \\
& =\operatorname{hofiber}\left(F\left(B \otimes_{A} \emptyset\right) \rightarrow \operatorname{holim}\left(S \in \mathcal{P}_{0}(\mathbf{n}+\mathbf{1}) \mapsto F\left(B \otimes_{A} S\right)\right)\right) \\
& \simeq \operatorname{hofiber}\left(F(A) \rightarrow T_{n} F(A)\right),
\end{aligned}
$$

as desired.

Lemma 6.11. If $F: \mathcal{C}_{f} \rightarrow \mathcal{S}$ is a degree $n$ functor, then $F(A) \rightarrow P_{n} F(A)$ is a weak equivalence. 
Proof. Since $F$ is degree $n, c r_{n+1} F \simeq \star$. Thus, $\perp_{n+1} F(A) \simeq \star$, and by Lemma 6.10. $F(A) \simeq T_{n} F(A)$. Moreover, since $T_{n}$ and $c r_{n+1}$ are both homotopy inverse limit constructions, and homotopy inverse limits commute,

$$
c r_{n+1} T_{n} F \simeq T_{n} c r_{n+1} F \simeq \star .
$$

Hence, $T_{n} F$ is also a degree $n$ functor and by Lemma 6.10

$$
T_{n} F(A) \simeq T_{n}^{2} F(A) .
$$

Continuing in this fashion, we see that

$$
F(A) \stackrel{\simeq}{\rightarrow} \operatorname{hocolim}_{k}\left(T_{n}^{k} F(A)\right)=P_{n} F(A) .
$$

With this, we prove Theorem 6.9

Proof. We prove this theorem by proving that we have a fibration sequence of spectra

$$
\left|\perp_{n+1}^{*+1} F(A)\right| \rightarrow F(A) \rightarrow P_{n} F(A) .
$$

Consider diagram (16). Following the same strategy as in the proof of Theorem 6.5 we see that it suffices to show that the rightmost vertical arrow is an equivalence and that the object in the bottom left corner is equivalent to $\star$. The first fact is a consequence of Lemma 6.11. To prove the second fact, we note that as was the case for Theorem 6.5, $P_{n}\left|\perp_{n+1}^{*+1} F\right| \simeq\left|P_{n} \perp_{n+1}^{*+1} F\right|$ since $F$ takes values in $\mathcal{S}$, and so it is enough to show that $P_{n} \perp_{n+1} F(A) \simeq \star$. This was done in Corollary 6.7. The result follows.

The proof of Theorem 6.9 relies on the critical observation in the proof of Lemma 6.10 that in order to obtain agreement between $\perp_{n+1} F$ and the fiber of $F \rightarrow T_{n} F$ we must evaluate at the same object over which the coproducts for $\perp_{n+1} F$ are taken. This suggests that for general $F$, the cotriple construction can be used in place of Goodwillie's construction only when evaluating at the initial object of the domain category. In fact, when evaluated at other objects, $\Gamma_{n} F$ and $P_{n} F$ can differ greatly.

Example 6.12. Consider the functor $H_{1}: \mathcal{U}_{*} \rightarrow \mathcal{S}$ that takes a space $X$ to the Eilenberg-Mac Lane spectrum associated to its first (singular) homology group. By Theorem 6.9. we know that $\Gamma_{1} H_{1}(\emptyset) \simeq P_{1} H_{1}(\emptyset)$. For an arbitrary space $X$, one can show that $\perp_{2} H_{1}(X) \simeq \star$, and hence, $\Gamma_{1} H_{1}(X) \simeq H_{1}(X)$. However, if $X$ is a connected space, then

$$
T_{1} H_{1}(X) \simeq \operatorname{holim}\left(H_{1}\left(* \otimes_{X}\{1\}\right) \rightarrow H_{1}\left(* \otimes_{X}\{1,2\}\right) \leftarrow H_{1}\left(* \otimes_{X}\{2\}\right)\right) \simeq \star
$$

since $* \otimes_{X}\{1\}$ and $* \otimes_{X}\{2\}$ are equivalent to the cone on $X$ and $* \otimes_{X}\{1,2\}$ is equivalent to the (unreduced) suspension of $X$. As a result, one sees that $P_{1} H_{1}(X) \simeq \star$ when $X$ is connected, so $\Gamma_{1} H_{1}$ and $P_{1} H_{1}$ are not equivalent as functors. One can obtain similar results for higher homology groups.

This example shows that we cannot guarantee in general that $\Gamma_{n} F$ and $P_{n} F$ agree as functors. However, we can show that the particular value $P_{n} F(X)$ is equivalent to the $n$th term in a cotriple Taylor tower for $F$, albeit not the same tower as used in Theorem 6.9. To do so, we change our focus from $\mathcal{C}_{f}$, which has a fixed initial and fixed terminal object, to the category $\mathcal{C}_{/ B}$ of objects over $B$. To state the result precisely we use the following notation. 
An object of the category $\mathcal{C}_{/ B}$ of objects over $B$ is a morphism $\beta: X \rightarrow B$ in $\mathcal{C}$. Given a functor $F: \mathcal{C}_{/ B} \rightarrow \mathcal{S}$, we can restrict it to the category $\mathcal{C}_{\beta}$ determined by objects that factor $\beta: X \rightarrow B$ in $\mathcal{C}$. We use $F^{\beta}$ to denote this restriction. We can define the cross effects of $F^{\beta}$ using $X$ as our initial object and $B$ as our final object. We denote these cross effects and the associated cotriples by $c r_{n}^{\beta} F$ and $\perp_{n}^{\beta} F$, respectively. Note that even if $\mathcal{C}_{/ B}$ had an initial object $A$ (so that it is secretly a category $\mathcal{C}_{f}$ for some fixed $\left.f: A \rightarrow B\right), c r_{n}^{\beta} F$ is not the restriction of $c r_{n} F$ to $\mathcal{C}_{\beta}$. For example, for an object $Z$ in $\mathcal{C}_{\beta}, c r_{2} F(Z, Z)$ is the total fiber of the diagram

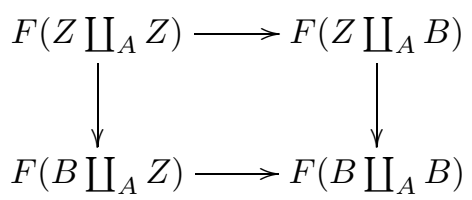

whereas $\mathrm{cr}_{2}^{\beta} F(Z, Z)$ is the total fiber of

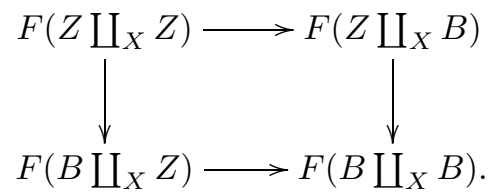

We use $\Gamma_{n}^{\beta} F$ to denote the homotopy cofiber of

$$
\left|\left(\perp_{n+1}^{\beta}\right)^{*+1} F\right| \rightarrow F .
$$

As indicated above, using the map $\beta$ in place of $f$ to define our cross effects changes the construction $\Gamma_{n} F$ to $\Gamma_{n}^{\beta} F$. However, this is not the case for $P_{n} F$, as its definition requires a specific initial object (the empty set, or the point in the base pointed case) and prohibits restriction to the category $\mathcal{C}_{\beta}$. In light of this, Theorem 6.9 can be restated to obtain equivalences between $\Gamma_{n}^{\beta} F(X)$ and $P_{n} F(X)$. Moreover, this equivalence is functorial in $X$.

Theorem 6.13. Let $F: \mathcal{C}_{/ B} \rightarrow \mathcal{S}$ and let $\beta: X \rightarrow B$ be an object in $\mathcal{C}_{/ B}$.

(1) There is a functor $\Gamma_{n}^{(-)} F: \mathcal{C}_{/ B} \rightarrow \mathcal{S}$ taking $\beta: X \rightarrow B$ to $\Gamma_{n}^{\beta} F(X)$.

(2) There is a natural weak equivalence

$$
\Gamma_{n}^{\beta} F(X) \simeq P_{n} F(X) .
$$

Proof. Let $\beta: X \rightarrow B$ and $\beta^{\prime}: Y \rightarrow B$ in $\mathcal{C}_{/ B}$. The functoriality of $\Gamma_{n}^{(-)} F$ comes from the fact that a map $\sigma: X \rightarrow Y$ in $\mathcal{C}_{/ B}$ induces enough maps between coproducts over $X$ and $Y$, respectively, to induce maps between $\perp_{n}^{\beta} F(X)$ and $\perp_{n}^{\beta^{\prime}} F(Y)$. The rest follows from Theorem 6.9.

\section{Convergence of the Cotriple tower}

This section identifies criteria that guarantee convergence of our tower. Our first goal is to show that our tower converges for analytic functors, just as Goodwillie's tower does. In the second part of this section we also identify conditions on cross effects that guarantee convergence. In this section we work with functors $F$ whose target category $\mathcal{S}$ is a category of spectra. Since analyticity requires a notion of connectivity in both the domain and target categories, we restrict ourselves to the 
setting of Goodwillie's calculus of homotopy functors for the results concerning analyticity. In particular, we let $\mathcal{T}$ denote the category of topological spaces and $\mathcal{T}_{g}$ denote the category determined by the morphism $g: C \rightarrow D$. For the results in the second part of this section, we will work with functors $F: \mathcal{C}_{f} \rightarrow \mathcal{S}$, where $\mathcal{C}$ is a simplicial model category and $\mathcal{C}_{f}$ is determined by a fixed morphism $f: A \rightarrow B$ in $\mathcal{C}$. Throughout this section we use $P_{\infty} F$ to denote the homotopy inverse limit of the Goodwillie tower for $F$ and $\Gamma_{\infty} F$ to denote the homotopy inverse limit of the cotriple tower. We say that the Goodwillie tower for $F$ converges at $X$ if the natural map $F(X) \rightarrow P_{\infty} F(X)$ is a weak equivalence. Convergence of the cotriple tower is defined analogously.

7.1. Analyticity and convergence. As in [9], by a $k$-connected map of spaces we mean a map whose homotopy fibers are all $(k-1)$-connected. Recall the following definitions from [9].

Definition 7.1 $([9,1.3])$. The $n$-cubical diagram $\mathcal{X}$ in $\mathcal{T}$ or $\mathcal{S}$ is $k$-cartesian provided that the map from $\mathcal{X}(\emptyset)$ to $\operatorname{holim}_{S \in \mathcal{P}_{0}(\mathbf{n})} \mathcal{X}(S)$ is $k$-connected.

Definition $7.2([9,4.1])$. A functor $F: \mathcal{T} \rightarrow \mathcal{S}$ is stably $n$-excisive, if the following is true for some numbers $c$ and $\kappa$ :

$E_{n}(c, \kappa):$ If $\mathcal{X}: \mathcal{P}(\mathbf{n}+\mathbf{1}) \rightarrow \mathcal{C}_{f}$ is any strongly cocartesian $(n+1)$-cube such that for all $s \in \mathbf{n}+\mathbf{1}$ the map $\mathcal{X}(\emptyset) \rightarrow \mathcal{X}(\{s\})$ is $k_{s}$-connected and $k_{s} \geq \kappa$, then the diagram $F(\mathcal{X})$ is $\left(-c+\sum k_{s}\right)$-cartesian.

Definition $7.3([9,4.2])$. A functor $F: \mathcal{T} \rightarrow \mathcal{S}$ is $\rho$-analytic if there is some number $q$ such that $F$ satisfies $E_{n}(n \rho-q, \rho+1)$ for all $n \geq 1$.

In 10, Goodwillie showed that his tower for $F$ converges at $X$ when $F$ is $\rho$ analytic and $X \rightarrow D$ is at least $(\rho+1)$-connected. We establish a similar result for our tower below, using the next lemma.

Lemma 7.4. If $F: \mathcal{T}_{g} \rightarrow \mathcal{S}$ is $\rho$-analytic and $\beta: X \rightarrow D$ is at least $(\rho+1)$ connected, then $\perp_{n+1}^{k} F(X)$ is at least $\left(q+(n+1)^{k}-1\right)$-connected. Here $q$ is the constant such that $F$ satisfies $E_{n}(n \rho-q, \rho+1)$.

Proof. Since $\perp_{n+1} F(X)$ is the total fiber of a strongly cocartesian $(n+1)$-cube in $\mathcal{T}_{g}, \perp_{n+1}^{k} F(X)$ can be described as the total fiber of a strongly cocartesian $(n+1)^{k}$-cube $\mathcal{Y}$. For each $S \in \mathcal{P}\left((\mathbf{n}+\mathbf{1})^{\mathbf{k}}\right), \mathcal{Y}(S)$ is a coproduct of copies of $X$ and $D$ over $C$ and each morphism is a coproduct of copies of $\beta$ and identity morphisms. In particular, this ensures that $\mathcal{Y}(\emptyset) \rightarrow \mathcal{Y}(\{s\})$ is at least $(\rho+1)$-connected for each $s \in(\mathbf{n}+\mathbf{1})^{\mathbf{k}}$. The analyticity condition then guarantees that the total fiber of $F(\mathcal{Y})$ is at least $\left(q+(n+1)^{k}-1\right)$-connected. The result follows.

Proposition 7.5. If $F: \mathcal{T}_{g} \rightarrow \mathcal{S}$ is $\rho$-analytic, satisfying $E_{n}(n \rho-q, \rho+1)$ for each $n$, and $X \rightarrow B$ is at least $(\rho+1)$-connected, then $F(X) \rightarrow \Gamma_{n} F(X)$ is at least $(q+n+1)$-connected. As a consequence, $F(X) \simeq \Gamma_{\infty} F(X)$.

Proof. By the lemma, we know that in each simplicial degree $\perp_{n+1}^{*+1} F(X)$ is at least $\left(q+(n+1)^{1}-1\right)$-connected. Since homotopy colimits preserve connectivity, it follows that the realization of $\perp_{n+1}^{*+1} F(X)$ is at least $\left(q+(n+1)^{1}-1\right)$-connected. The result follows using the fact that in spectra,

$$
\left|\perp_{n+1}^{*+1} F(X)\right| \rightarrow F(X) \rightarrow \Gamma_{n} F(X)
$$

is also a fibration sequence. 
7.2. Cross effects and convergence. Mimicking the convergence results for the abelian case found in section 4 of [13, we can place conditions on $\perp_{n+1} F$ that guarantee convergence of the cotriple tower.

Proposition 7.6. Let $F: \mathcal{C}_{f} \rightarrow \mathcal{S}$ be a functor that takes values in connective spectra. Let $X$ be an object in $\mathcal{C}_{f}$. Suppose that there is a $c \geq 0$ such that for $1 \leq t \leq c+1, \perp_{n+1}^{t} F(X)$ is at least $(c+1-t)$-connected. Then $\gamma_{n} F: F(X) \rightarrow$ $\Gamma_{n} F(X)$ is $(c+1)$-connected.

Proof. The connectivity condition is sufficient to ensure that $\left|\perp_{n+1}^{*+1} F(X)\right|$ is at least $c$-connected. To see this one can use the spectral sequence associated to the simplicial spectrum $\perp_{n+1}^{*+1} F(X)$ that has

$$
E_{p, q}^{1}=\pi_{p}\left(\perp_{n+1}^{q+1} F(X)\right)
$$

and converges to $\pi_{p+q}\left(\left|\perp_{n+1}^{*+1} F(X)\right|\right)$ (see, e.g., 4] for details). The connectivity condition guarantees that $E_{p, q}^{1} \cong 0$ for $p+q \leq c$. Hence, $\left|\perp_{n+1}^{*+1} F(X)\right|$ must be at least $c$-connected. The result follows again by using the fibration sequence

$$
\left|\perp_{n+1}^{*+1} F(X)\right| \rightarrow F(X) \rightarrow \Gamma_{n} F(X) .
$$

The condition that $\perp_{n+1}^{t} F(X)$ is at least $(c+1-t)$-connected for $1 \leq t \leq c$ is like that of stable $n$-excision in Goodwillie's calculus, in that the condition guarantees a certain connectivity of the map $F(X) \rightarrow \Gamma_{n} F(X)$. We use this condition to guarantee convergence of our tower as follows.

Definition 7.7. For a functor $F: \mathcal{C}_{f} \rightarrow \mathcal{S}$, object $X$ in $\mathcal{C}_{f}$, and $n \geq 0$, we set

$$
F_{\text {con }}(X, n)=\max \left\{c \in \mathbb{Z} \mid \operatorname{conn}\left(\perp_{n+1}^{t} F(X)\right) \geq c+1-t \text { for } 1 \leq t \leq c+1\right\} .
$$

With this, the next proposition is an immediate consequence of Proposition 7.6.

Proposition 7.8. Let $F: \mathcal{C}_{f} \rightarrow$ Spec be a functor that takes values in connective spectra. If $\lim _{n \rightarrow \infty} F_{\text {con }}(X, n)=N$, then $F(X) \rightarrow \Gamma_{\infty} F(X)$ is $(N+1)$-connected. When $N=\infty$, the cotriple tower converges for $X$.

\section{Appendix A. Proofs of HOMOTOPy LIMIT PROPERTIES}

In this appendix, we address the four properties of homotopy inverse limits in Lemma 2.5 that are essential to our proof in section 3 that $t$ is a cotriple. Although these properties are widely accepted as being true, we had difficulty finding proofs of them in the literature. As they are critical to our work in section 3 , we include their proofs in this appendix. For $\mathscr{C}$ a category, let $\operatorname{hom}_{\mathscr{C}}(A, B)$ be the set of morphisms between $A, B \in \mathrm{ob}(\mathscr{C})$. Recall that a simplicial model category also has a simplicial set of maps. For objects $A$ and $B$, we use $\operatorname{Hom}_{\mathscr{C}}(A, B)$ to denote this and note that $\left(\operatorname{Hom}_{\mathscr{C}}(A, B)\right)_{n}:=\operatorname{hom}_{\mathscr{C}}(A \otimes \Delta[n], B)$.

Let $\mathcal{M}$ be a simplicial model category and let $\mathcal{C}$ be a small category. Following Hirschhorn [11, for $X$ a $\mathcal{C}$-diagram in $\mathcal{M}$ and $K$ a $\mathcal{C}$-diagram in simplicial sets, define

$$
\operatorname{hom}^{\mathcal{C}}(K, X)=\text { equalizer }\left(\prod_{C \in \mathcal{C}}\left(X_{C}\right)^{K(C)} \longrightarrow \prod_{f: C \rightarrow C^{\prime} \in \mathcal{C}}\left(X_{C^{\prime}}\right)^{K(C)}\right),
$$


where $K(C)$ is an object in $S S$, the category of simplicial sets. The above makes use of the fact that for $M \in \mathrm{ob}(\mathcal{M})$ and $K \in \mathrm{ob}(S S)$, there is an object $M^{K}$ in $\mathcal{M}$.

When we let $K=N_{\bullet}(\mathcal{C} \downarrow *)$ (the classifying space or nerve of the over category $\mathcal{C} \downarrow *$; see [11, 14.1.1]), we define

$$
\operatorname{holim}_{\mathcal{C}} X=\operatorname{hom}^{\mathcal{C}}\left(N_{\bullet}(\mathcal{C} \downarrow *), X(*)\right) .
$$

As noted on page 379 of [11, this model for $\operatorname{holim}_{\mathcal{C}} X$ is homotopy invariant only when $X$ is objectwise fibrant. When $X$ is objectwise fibrant, by Corollary 18.5.2(2) of [1], $\operatorname{holim}_{\mathcal{C}} X$ is a fibrant object of $\mathcal{M}$.

To establish the desired homotopy inverse limit properties, we make use of the following important adjunction. This adjunction is stated and proved for morphism sets in [11, but it is straightforward to generalize the proof to simplicial mapping spaces to obtain the version stated below.

Proposition 18.3.10(2) of [11. Let $X$ be a $\mathcal{C}$-diagram in $\mathcal{M}, K$ a $\mathcal{C}$-diagram of simplicial sets and let $W$ be an object of $\mathcal{M}$. Then there is a natural isomorphism

$$
\operatorname{Hom}_{\mathcal{M}}\left(W, \operatorname{hom}^{\mathcal{C}}(K, X)\right) \cong \operatorname{Hom}_{S S^{\mathcal{C}}}\left(K, \operatorname{Hom}_{\mathcal{M}}(W, X)\right) .
$$

Here $S S$ is the category of simplicial sets and $S S^{\mathcal{C}}$ is the category of $\mathcal{C}$-diagrams in simplicial sets.

With this adjunction and the Yoneda Lemma, we can establish the desired properties for this model of holim in $\mathcal{M}$ by reducing them to properties of diagrams of simplicial sets. In order to do this, we first recall some facts about diagrams of simplicial sets.

By Definition 18.2.3(1) and Proposition 18.2.5 of [11, for $A$ and $B$ elements of $S S^{\mathcal{C}}, \operatorname{Hom}_{S S^{\mathcal{C}}}(A, B)$ consists of simplicial sets of the form

$$
[n] \mapsto \operatorname{Nat}_{S S^{C}}(A \otimes \Delta[n], B) .
$$

That is, the $n$-simplices are simplicial maps from $A(C) \times \Delta[n]$ to $B(C)$ that are natural in $\mathcal{C}$. Since the one point set $*$ is terminal, we see that the constant $\mathcal{C}$ diagram to $*$ is terminal in $S S^{\mathcal{C}}$.

Given a functor $\alpha$ from $\mathcal{D}$ to $\mathcal{C}$ of small categories and objects $A, B$ in $S S^{\mathcal{C}}$, we have a natural map

$$
\operatorname{Hom}_{S S^{\mathcal{C}}}(A, B) \stackrel{\left.\right|_{\alpha}}{\longrightarrow} \operatorname{Hom}_{S S^{\mathcal{D}}}(A \circ \alpha, B \circ \alpha)
$$

by restricting $f$ to $\alpha(\mathcal{C})$,or $\left.f\right|_{\alpha}(D)=f(\alpha(D))$.

The last property we want is a generalization of the Exponential Law found in [7, Proposition I.5.1, to simplicial mapping spaces for diagrams of simplicial sets. Given $X \in \mathrm{ob}\left(S S^{\mathcal{D}}\right)$ and $Y \in \mathrm{ob}\left(S S^{\mathcal{C} \times \mathcal{D}}\right)$ there is an evaluation map in $S S^{\mathcal{C} \times \mathcal{D}}$,

$$
\operatorname{Hom}_{S S^{\mathcal{D}}}(X(\star), Y(-, \star)) \times X \stackrel{e v}{\longrightarrow} Y,
$$

given by sending $\left(f, x_{n}\right) \in\left(\operatorname{Hom}_{S S^{\mathcal{D}}}(X(\star), Y(-, \star)) \times X\right)_{n}$ to $f\left(x_{n}, \operatorname{id}_{n}\right)$. Using this we obtain the following.

Proposition [Exponential Law]. For objects $K$ in $S S^{\mathcal{C}}, X$ in $S S^{\mathcal{D}}$ and $Y$ in $S S^{\mathcal{C} \times \mathcal{D}}$, there is a natural isomorphism

$$
\operatorname{Hom}_{S S^{\mathcal{C}}}\left(K(*), \operatorname{Hom}_{S S^{\mathcal{D}}}(X(\star), Y(*, \star))\right) \stackrel{e v_{*}}{\longrightarrow} \operatorname{Hom}_{S S^{\mathcal{C}} \times \mathcal{D}}(K(*) \times X(\star), Y(*, \star)) .
$$


Proof. The function $e v_{*}$ is defined by sending $g: K \times \Delta[n] \rightarrow \operatorname{Hom}_{S S^{\mathcal{D}}}(X, Y)$ to the composite

$$
K \times X \times \Delta[n] \cong K \times \Delta[n] \times X \stackrel{g \times 1}{\longrightarrow} \operatorname{Hom}_{S S^{\mathcal{D}}}(X, Y) \times X \stackrel{e v}{\longrightarrow} Y .
$$

This is an isomorphism whose inverse is the map defined by sending $g: K \times X \times$ $\Delta[n] \rightarrow Y$ to the map $g_{*}: K \times \Delta[n] \rightarrow \operatorname{Hom}_{S S^{\mathcal{D}}}(X, Y)$, where $g_{*}$ sends

$$
x \in K_{m} \times \Delta[n]_{m} \cong\left(\Delta[m] \stackrel{i_{(x \times \tau)}}{\longrightarrow} K \times \Delta[n]\right)
$$

to the composite

$$
X \times \Delta[m] \stackrel{1 \times i_{(x \times \tau)}}{\longrightarrow} X \times K \times \Delta[n] \cong K \times X \times \Delta[n] \stackrel{g}{\longrightarrow} Y .
$$

Here $\iota_{x \times \tau}$ is as defined on p. 6 of [7].

We now establish the four properties of our model for holim $\mathcal{C} X$ for $\mathcal{M}$ that we need to prove $t$ is a cotriple, that is, we prove the first four properties of Lemma 2.5 .

Property 1. Given $\mathcal{C}$ and $\mathcal{D}$ small categories and $X$ a $\mathcal{C} \times \mathcal{D}$ diagram in $\mathcal{M}$, there are natural isomorphisms

$$
\operatorname{holim}_{\mathcal{C}} \operatorname{holim}_{\mathcal{D}} X \cong \operatorname{holim}_{\mathcal{C} \times \mathcal{D}} X \cong \operatorname{holim}_{\mathcal{D}} \operatorname{holim}_{\mathcal{C}} X \text {. }
$$

Proof. Recall that $N_{\bullet}(\mathcal{C} \times \mathcal{D} \downarrow *) \cong N_{\bullet}(\mathcal{C} \downarrow *) \times N_{\bullet}(\mathcal{D} \downarrow *)$ as simplicial sets. Making repeated use of the two propositions, we obtain the following sequences of isomorphisms of simplicial sets:

$$
\begin{aligned}
\operatorname{Hom}_{\mathcal{M}}\left(W, \operatorname{holim}_{\mathcal{C} \times \mathcal{D}} X\right) & =\operatorname{Hom}_{\mathcal{M}}\left(W, \operatorname{hom}^{\mathcal{C} \times \mathcal{D}}\left(N_{\bullet}(\mathcal{C} \times \mathcal{D} \downarrow *), X\right)\right) \\
& \cong \operatorname{Hom}_{S S^{\mathcal{C}} \times \mathcal{D}}\left(N_{\bullet}(\mathcal{C} \times \mathcal{D} \downarrow *), \operatorname{Hom}_{\mathcal{M}}(W, X)\right) \\
& \cong \operatorname{Hom}_{S S^{\mathcal{C}} \times \mathcal{D}}\left(N_{\bullet}(\mathcal{C} \downarrow *) \times N_{\bullet}(\mathcal{D} \downarrow *), \operatorname{Hom} \mathcal{M}(W, X)\right) \\
& \cong \operatorname{Hom}_{S S^{\mathcal{C}}}\left(N_{\bullet}(\mathcal{C} \downarrow *), \operatorname{Hom}_{S S^{\mathcal{D}}}\left(N_{\bullet}(\mathcal{D} \downarrow *), \operatorname{Hom}(W, X)\right)\right) \\
& \cong \operatorname{Hom}_{S S^{\mathcal{C}}}\left(N_{\bullet}(\mathcal{C} \downarrow *), \operatorname{Hom}_{\mathcal{M}}\left(W, \operatorname{hom}^{\mathcal{D}}\left(N_{\bullet}(\mathcal{D} \downarrow *), X\right)\right)\right) \\
& \cong \operatorname{Hom}_{\mathcal{M}}\left(W, \operatorname{hom}^{\mathcal{C}}\left(N_{\bullet}(\mathcal{C} \downarrow *), \operatorname{hom}^{\mathcal{D}}\left(N_{\bullet}(\mathcal{D} \downarrow *), X\right)\right)\right) \\
& \cong \operatorname{Hom}_{\mathcal{M}}\left(W, \operatorname{holim}_{\mathcal{C}} \operatorname{holim}_{\mathcal{D}} X\right) .
\end{aligned}
$$

By the Yoneda Lemma, we obtain the natural isomorphism.

Property 2. If $\alpha: \mathcal{C} \rightarrow \mathcal{D}$ is a functor of small categories, and $X$ is a $\mathcal{D}$-diagram in $\mathcal{M}$, we obtain a natural map

$$
\operatorname{holim}_{\mathcal{D}} X \rightarrow \operatorname{holim}_{\mathcal{C}}(X \circ \alpha)
$$

Proof. Observe that there is a natural transformation of objects in $S S^{\mathcal{C}}$ :

$$
N_{\bullet}(\mathcal{C} \downarrow *) \stackrel{\alpha}{\longrightarrow} N_{\bullet}(\mathcal{D} \downarrow *) \circ \alpha .
$$


Then

$$
\begin{aligned}
\operatorname{Hom}_{\mathcal{M}}\left(W, \operatorname{holim}_{\mathcal{D}} X\right) & \cong \operatorname{Hom}_{S S^{\mathcal{D}}}\left(N_{\bullet}(\mathcal{D} \downarrow *), \operatorname{Hom}_{\mathcal{M}}(W, X)\right) \\
& \rightarrow \operatorname{Hom}_{S S^{\mathcal{C}}}\left(N_{\bullet}(\mathcal{D} \downarrow *) \circ \alpha, \operatorname{Hom}_{\mathcal{M}}(W, X) \circ \alpha\right) \\
& \stackrel{\alpha^{*}}{\longrightarrow} \operatorname{Hom}_{S S^{\mathcal{C}}}\left(N_{\bullet}(\mathcal{C} \downarrow *), \operatorname{Hom}_{\mathcal{M}}(W, X) \circ \alpha\right) \\
& \cong \operatorname{Hom}_{S S^{\mathcal{C}}}\left(N_{\bullet}(\mathcal{C} \downarrow *), \operatorname{Hom}_{\mathcal{M}}(W, X \circ \alpha)\right) \\
& \cong \operatorname{Hom}_{\mathcal{M}}\left(W, \operatorname{hom}^{\mathcal{C}}(N \bullet(\mathcal{C} \downarrow *), X \circ \alpha)\right) \\
& \cong \operatorname{Hom}_{\mathcal{M}}\left(W, \operatorname{holim}_{\mathcal{C}} X \circ \alpha\right),
\end{aligned}
$$

and so by Yoneda, we have our natural transformation.

Property 3. If $T$ is a constant $\mathcal{C}$-diagram in $\mathcal{M}$ with $T(C)=T$ and $T(f)=\mathrm{id}_{T}$ where $T$ is a terminal object of $\mathcal{M}$, then

$$
\operatorname{holim}_{\mathcal{C}} T \cong T \text {. }
$$

Proof.

$$
\begin{aligned}
\operatorname{Hom}_{\mathcal{M}}\left(W, \operatorname{holim}_{\mathcal{C}} T\right) & \cong \operatorname{Hom}_{S S^{\mathcal{C}}}\left(N_{\bullet}(\mathcal{C} \downarrow *), \operatorname{Hom}_{\mathcal{M}}(W, T)\right) \\
& \cong *
\end{aligned}
$$

(this is because $\operatorname{Hom}_{\mathcal{M}}(W, T)=*$, the constant one point $\mathcal{C}$-diagram of simplicial sets, since $T$ is terminal in $\mathcal{M})$. Since $W$ was arbitrary, $\operatorname{holim}_{\mathcal{C}} T$ is terminal and terminal objects are all isomorphic.

Property 4. If $\mathcal{I}$ is the trivial category on $i$, then for $X$ any $\mathcal{I}$-diagram in $\mathcal{M}$,

$$
\operatorname{holim}_{\mathcal{I}} X \cong X(i) \text {. }
$$

Proof. This is Proposition 18.3.7(2) of [1].

\section{Appendix B. by Rosona Eldred}

Lemma 6.10 may be viewed as the base case of a more general phenomenon. The purpose of this appendix is to explain how $\perp_{n+1}^{*+1} F$ and the tower of functors $\mathrm{T}_{n}^{k} F$ defining $\mathrm{P}_{n} F$ are related by a sequence of fibration sequences. We prove the following proposition.

Proposition B.1. For a functor $F: \mathcal{C}_{f} \rightarrow \mathcal{S}$, there is a homotopy fiber sequence $\left|\operatorname{sk}_{k}\left(\perp_{n+1}^{*+1} F\right)(A)\right| \rightarrow F(A) \rightarrow \mathrm{T}_{n}^{k+1} F(A)$.

This gives an alternative approach to understanding Theorem 6.9. since as $k$ goes to infinity, Proposition B.1 suggests that there is a homotopy fiber sequence $\left|\perp_{n+1}^{*+1} F(A)\right| \rightarrow F(A) \rightarrow P_{n} F(A)$, i.e., the result of Theorem 6.9.

The proof of Proposition B.1 is by induction, taking Lemma 6.10 as the base case with $k=0$.

B.1. Lemmas necessary for proof. Given a square of spectra,

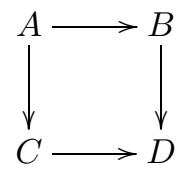


we have an associated diagram which includes the fibers. Then, let

$$
\begin{array}{rlrl}
h_{1} & :=\operatorname{hofib}(C \rightarrow D) & & h_{4}:=\operatorname{hofib}(A \rightarrow C) \\
h_{2} & :=\operatorname{hofib}(A \rightarrow B) & h_{5}:=\operatorname{hofib}(B \rightarrow D) \\
h_{3} & :=\operatorname{hofib}\left(h_{4} \rightarrow h_{5}\right) & h_{6}:=\operatorname{hofib}(A \rightarrow D) . \\
& =\operatorname{hofib}\left(h_{2} \rightarrow h_{1}\right) & &
\end{array}
$$

Lemma B.2. With the definitions given above, the following is a cartesian square:

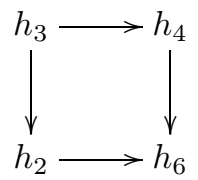

Proof of Lemma B.2. We can construct an associated diagram of fibers. It is the leftmost in the homotopy fiber sequence shown in Figure 1.

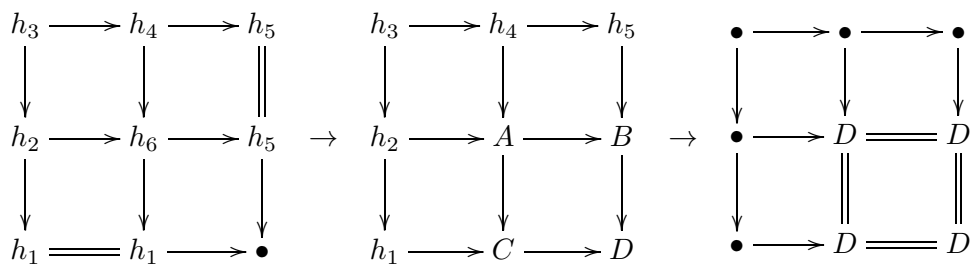

Figure 1. Homotopy fiber sequence of diagrams

Since $h_{3}=\operatorname{hofiber}\left(h_{4} \rightarrow h_{5}\right)$, then $h_{3} \rightarrow h_{4} \rightarrow h_{5}$ is a homotopy fiber sequence. This says that $\Omega h_{5}=\operatorname{hofiber}\left(h_{3} \rightarrow h_{4}\right)$. Figure 2 illustrates that $h_{2} \rightarrow h_{6} \rightarrow h_{5}$ is also a homotopy fiber sequence, as homotopy-fiber-taking commutes.

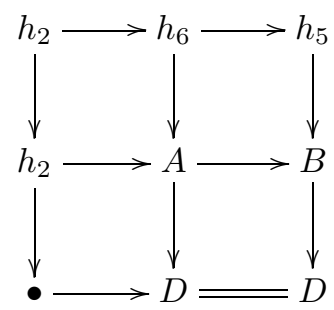

Figure $2 . h_{2} \rightarrow h_{6} \rightarrow h_{5}$ is a homotopy fiber sequence

Thus, $\Omega h_{5} \rightarrow h_{2} \rightarrow h_{6}$ is also a homotopy fiber sequence. We can extend the square to the left with its homotopy fibers, as in Figure 3. As they are equivalent and we're in spectra (which cures the basepoint troubles), we can conclude that the square is a homotopy pullback.

Lemma B.3. $\left|\operatorname{sk}_{k} \perp_{n+1}^{*+1} F\right| \simeq \operatorname{hocolim}_{\mathscr{P}_{0}^{o p}([k])}\left(\perp_{n+1}^{*+1} F\right)$. 


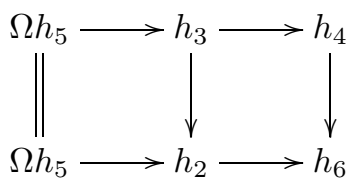

Figure 3. Square and its fibers

Proof of Lemma B.3. Let $\# S$ be the cardinality of the set $S$. From [21, we know the natural functor that maps $\mathscr{P}_{0}([n])$ to $\Delta_{\leq n}$ via $S \mapsto[\# S-1]$ is homotopy left cofinal. Its dual which maps $\mathscr{P}_{0}([n])^{o p}$ to $\Delta_{\leq n}^{o p}$ is then homotopy right cofinal. That is, we have that hocolim ${ }_{\Delta_{\leq n}^{o p}} X_{\bullet} \simeq \operatorname{hocolim}_{\mathscr{P}_{0}([n])^{o p} X_{\bullet}}$.

Given that $\left|\operatorname{sk}_{n} X_{\bullet}\right| \simeq$ hocolim $_{\Delta_{\leq n}^{o p}} X_{\bullet}$, with $X_{\bullet}=\perp_{n+1}^{*+1} F$, we have the statement of our lemma.

Lemma B.4. For $F: \mathcal{C}_{f} \rightarrow \mathcal{S}$,

$\left|\operatorname{sk}_{k} \perp_{n+1}^{*+1} F\right| \simeq \operatorname{hocolim}\left(\left|\operatorname{sk}_{k-1} \perp_{n+1}^{*+1} F\right| \leftarrow\left|\operatorname{sk}_{k-1} \perp_{n+1}^{*+1}\left(\perp_{n+1} F\right)\right| \rightarrow_{n+1} F\right)$.

Note that Lemma B.4 is a simple application of the covering lemma for hocolim cubes (given on p. 299 of [9] as the dual situation to Proposition 0.2 in the same paper) to the $(k+1)$-cube that at $U \in \mathscr{P}([k])$ is $\perp_{n+1}^{|[k]-U|} F$, whose co-punctured (with the final element of the cube removed) hocolim is equivalent to $\left|\operatorname{sk}_{k} \perp_{n+1}^{*+1} F\right|$ by Lemma B.3.

B.2. General case of induction. Assume that $\left|\operatorname{sk}_{k-1}\left(\perp_{n+1}^{*+1} F(A)\right)\right| \rightarrow F(A) \rightarrow$ $\mathrm{T}_{n}^{k} F(A)$ is a homotopy fiber sequence. Then construct the diagram of homotopy fiber sequences in Figure 4.

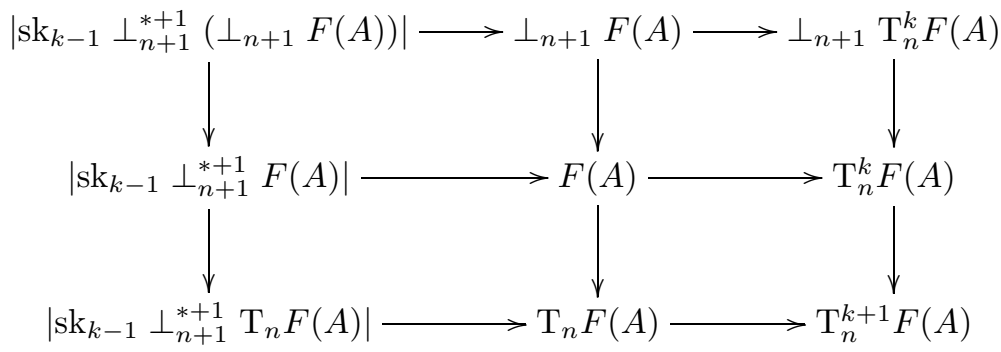

FigURE $4 . \perp_{n+1}$ and iterated fiber diagram

Since homotopy limit constructions commute, $\perp_{n+1} \mathrm{~T}_{n}^{k} F(A) \simeq \mathrm{T}_{n}^{k} \perp_{n+1} F(A)$, and the top line is also a homotopy fiber sequence.

The application of Lemma B.2 to the diagram of Figure 4 lets us conclude that Figure 5 is a cartesian square. Since we're in spectra, we also know that it is cocartesian.

We now apply Lemma B.4 to conclude that hofib $\left(F(A) \rightarrow \mathrm{T}_{n}^{k+1} F(A)\right) \simeq \mid \operatorname{sk}_{k} \perp_{n+1}^{*+1}$ $F(A) \mid$. 


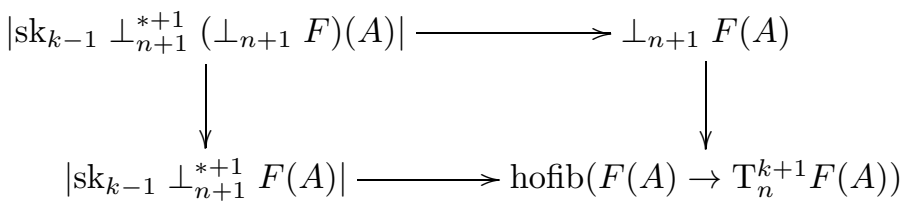

FiguRE 5. Desired cartesian square for general $k$

\section{ACKNOWLEDGMENTS}

The authors were able to meet and work together several times during the writing of this paper because of the generosity and hospitality of the following: the Midwest Topology Network (funded by NSF grant DMS-0844249), the Union College Faculty Research Fund, the Pacific Institute for the Mathematical Sciences, and the mathematics department of the University of Illinois at Urbana-Champaign. We thank them for their support. We thank Rosona Eldred, Agnès Beaudry, Mona Merling, and Sarah Yeakel for their assistance in confirming the homotopy limit properties of Lemma 2.5. We also thank Tom Goodwillie for the body of work that inspired this paper, and for the understanding of the calculus of functors that he has imparted to us over the years. Finally, we thank the anonymous referee for a very careful and thoughtful review. The referee's comments and corrections improved this paper substantially. In particular, the referee identified a critical error in an earlier version of section 3, and the suggestion that we prove $t$ is a cotriple directly and use the category of $t$-coalgebras was crucial in resolving this problem.

\section{REFERENCES}

[1] A. K. Bousfield and D. M. Kan, Homotopy limits, completions and localizations, Lecture Notes in Mathematics, Vol. 304, Springer-Verlag, Berlin-New York, 1972. MR0365573 (51 \#1825)

[2] Wojciech Chachólski and Jérôme Scherer, Homotopy theory of diagrams, Mem. Amer. Math. Soc. 155 (2002), no. 736, x+90, DOI 10.1090/memo/0736. MR.1879153 (2002k:55026)

[3] Boris Chorny and William G. Dwyer, Homotopy theory of small diagrams over large categories, Forum Math. 21 (2009), no. 2, 167-179, DOI 10.1515/FORUM.2009.009. MR2503302 (2010a:55022)

[4] D. Dugger, A primer on homotopy colimits, preprint, 2013. http://pages.uoregon.edu/ ddugger/hocolim.pdf

[5] Samuel Eilenberg and Saunders Mac Lane, On the groups H(П, n). II. Methods of computation, Ann. of Math. (2) 60 (1954), 49-139. MR0065162 (16,391a)

[6] A. D. Elmendorf, I. Kriz, M. A. Mandell, and J. P. May, Rings, modules, and algebras in stable homotopy theory, Mathematical Surveys and Monographs, vol. 47, American Mathematical Society, Providence, RI, 1997. With an appendix by M. Cole. MR.1417719 (97h:55006)

[7] Paul G. Goerss and John F. Jardine, Simplicial homotopy theory, Progress in Mathematics, vol. 174, Birkhäuser Verlag, Basel, 1999. MR1711612 (2001d:55012)

[8] Paul Goerss and Kristen Schemmerhorn, Model categories and simplicial methods, Interactions between homotopy theory and algebra, Contemp. Math., vol. 436, Amer. Math. Soc., Providence, RI, 2007, pp. 3-49, DOI 10.1090/conm/436/08403. MR2355769 (2009a:18010)

[9] Thomas G. Goodwillie, Calculus. II. Analytic functors, K-Theory 5 (1991/92), no. 4, 295332, DOI 10.1007/BF00535644. MR1162445 (93i:55015)

[10] Thomas G. Goodwillie, Calculus. III. Taylor series, Geom. Topol. 7 (2003), 645-711 (electronic), DOI 10.2140/gt.2003.7.645. MR2026544(2005e:55015) 
[11] Philip S. Hirschhorn, Model categories and their localizations, Mathematical Surveys and Monographs, vol. 99, American Mathematical Society, Providence, RI, 2003. MR.1944041 (2003j:18018)

[12] Mark Hovey, Brooke Shipley, and Jeff Smith, Symmetric spectra, J. Amer. Math. Soc. 13 (2000), no. 1, 149-208, DOI 10.1090/S0894-0347-99-00320-3. MR.1695653 (2000h:55016)

[13] B. Johnson and R. McCarthy, Deriving calculus with cotriples, Trans. Amer. Math. Soc. 356 (2004), no. 2, 757-803 (electronic), DOI 10.1090/S0002-9947-03-03318-X. MR2022719 (2005m:18016)

[14] Nicholas J. Kuhn, Goodwillie towers and chromatic homotopy: an overview, Proceedings of the Nishida Fest (Kinosaki 2003), Geom. Topol. Monogr., vol. 10, Geom. Topol. Publ., Coventry, 2007, pp. 245-279, DOI 10.2140/gtm.2007.10.245. MR.2402789 (2009h:55009)

[15] Saunders MacLane, Categories for the working mathematician, Springer-Verlag, New YorkBerlin, 1971. Graduate Texts in Mathematics, Vol. 5. MR0354798 (50 \#7275)

[16] Andrew John Mauer-Oats, Algebraic Goodwillie calculus and a cotriple model for the remainder, Trans. Amer. Math. Soc. 358 (2006), no. 5, 1869-1895 (electronic), DOI 10.1090/S00029947-05-03936-X. MR2197433 (2006i:55015)

[17] Andrew John Mauer-Oats, Goodwillie calculi, ProQuest LLC, Ann Arbor, MI, 2002. Thesis (Ph.D.)-University of Illinois at Urbana-Champaign. MR2704008

[18] Daniel G. Quillen, Homotopical algebra, Lecture Notes in Mathematics, No. 43, SpringerVerlag, Berlin-New York, 1967. MR0223432 (36 \#6480)

[19] C. L. Reedy, Homotopy theory of model categories, preprint 1973.

[20] M. Shulman, Homotopy limits and colimits and enriched category theory, preprint.

[21] Dev P. Sinha, The topology of spaces of knots: cosimplicial models, Amer. J. Math. 131 (2009), no. 4, 945-980, DOI 10.1353/ajm.0.0061. MR2543919(2010m:57033)

[22] Charles A. Weibel, An introduction to homological algebra, Cambridge Studies in Advanced Mathematics, vol. 38, Cambridge University Press, Cambridge, 1994. MR1269324|(95f:18001)

Department of Mathematics and Statistics, University of Calgary, 2500 University

Drive NW, Calgary, Alberta T2N 1N4, Canada

E-mail address: bauerk@ucalgary.ca

Department of Mathematics, Union College, 807 Union Street, Schenectady, New YORK 12308

E-mail address: johnsonb@union.edu

Department of Mathematics, University of Illinois at Urbana-Champaign, 1409 W. Green Street, Urbana, Illinois 61801-2907

E-mail address: rmccrthy@illinois.edu

Mathematisches Institut, University of Muenster, Einsteinstrasse 62, 48149 Münster, GERMANY

E-mail address: eldred@uni-muenster.de 